\title{
OPTIMAL LASER HEATING OF PLASMAS CONFINED IN STRONG SOLENOIDAL MAGNETIC FIELDS *
}

\author{
Javier VITELA E. \\ Centro de Estudios Nucleares, Universidad Nacional Autónoma de México, 04510 México, D.F.
}

\author{
A. Ziya AKCASU \\ Department of Nuclear Engineering, The University of Michigan, Ann Arbor, MI 48I09, USA
}

Received October 1985

\begin{abstract}
Optimal Control Theory is used to analyze the laser-heating of plasmas confined in strong solenoidal magnetic fields. Heating strategies that minimize a linear combination of heating time and total energy spent by the laser system are found. A numerical example is used to illustrate the theory. Results of this example show that by an appropriate modulation of the laser intensity, significant savings in the laser energy are possible with only slight increases in the heating time. However, results may depend strongly on the initial state of the plasma and on the final ion temperature.
\end{abstract}

\section{Introduction}

The optimal laser heating of plasma ions from an initial temperature to a desired final temperature using Optimal Control Theory has been discussed for a particular case by Vagners et al. [1]. They considered the problem of Minimum Heating Time of a plasma confined in a strong solenoidal magnetic field, where thermal conductivity and particle diffusion in both radial and axial directions are neglected. They neglected bremsstrahlung losses and the work done by the plasma against the magnetic field during compression or expansion. In addition they constrained the total energy available from the laser and determined the optimal laser intensity profile that minimize the heating time for only one set of initial conditions.

In this paper we generalize their work by including bremsstrahlung losses. We do not constraint the total laser energy available since this leads to cases with no solution, instead we minimize a linear combination of heating time and total energy spent with appropriate weighting coefficients. By adjusting the latter we obtain a set of heating strategies ranging from minimum heating time to minimum heating energy.

The physical model we consider here is of course limited, further extensions like those including electron heat conduction and plasma losses out the ends are certainly desirable, however the complexity of the dynamical description of the system quickly becomes unmanageable. Thus, a compromise is needed between the completeness of the physical model and the complexity of the calculations of the optimization problem.

The solution of our problem requires the use of optimal control theory [2]. This theory yields necessary conditions in the form of a two point boundary value problem. In this work we have succeeded in carrying out the optimization calculations to the end by developing a "Flooding" technique. This technique permits us to obtain the solution for any set of initial conditions, i.e., any initial values of the electron and ion temperatures.

Within the limitations of the plasma model adopted in this paper, it will be shown that considerable

* Based on a thesis submitted as partial fulfillment for a Ph.D. degree at The University of Michigan. 
savings can be achieved in the total laser energy requirement by resorting to laser intensity optimization.

The optimization problem arises from the fact that the laser energy initially heats the electrons, causing an increase in the electron temperature. Overheating the electrons reduces the electron-ion energy transfer rate and the coupling of the laser beam to the electrons while increasing radiation losses. This suggests heating strategies for example, in which electron-ion energy transfer rate is maximized in order to minimize the heating time, or perhaps one wants to minimize the total energy supplied by the laser system that heats the ions to a specified final temperature.

\section{Description of the physical model}

The physical model we consider here is that of a fully ionized neutral plasma, confined in an axially uniform magnetic field. Particle diffusion and ohmic heating are neglected under the assumptions of a large electrical conductivity and a large solenoidal magnetic field. In addition plasma losses from the ends and electron thermal conduction are not included in the model $[1,3]$.

The plasma density and temperatures are assumed uniform in space but time varying, due to the compression or expansion of the plasma column. The work done by the plasma column during these compressions and expansions is also neglected. It is assumed that quasistatic pressure exists at any time along the plasma column.

The plasma is heated uniformly by a laser beam propagating along the direction of the magnetic field [4]. The laser energy is mainly absorbed by the electrons by the absorption mechanism known as inverse bremsstrahlung [5].

As the electrons absorb energy from the radiation field they transfer energy to the ions through collisions. Assuming that both electrons and ions have Maxwellians distributions at different temperatures, the energy transfer takes place at the classical equipartition rate [6].

The energy losses included in the model are those of bremsstrahlung radiation [7]. Although these losses are not important at low electron temperatures they play a significant role when higher temperatures are attained by electrons during heating. The laser frequency is taken to be greater than the plasma frequency so that reflection losses at the vacuum plasma boundary are minimal. When the layers of the plasma column are heated, the laser absorption coefficient decreases; hence, the layers become more transparent to the incident radiation, allowing it to penetrate deeper in the plasma. This phenomena gives rise to what has been called a "bleaching wave" [4], as the laser radiation burns its way along the plasma column. It was shown by Yuen et al. [4], that for a constant laser pulse a sharp boundary between the hot and cold regions of the plasma is defined. As far as the hot region is concerned the electron and ion temperature remain approximately constant in space with values corresponding to their values at the boundary. Thus, it is reasonable to expect that the temporal behavior of the electron and ion temperatures at the boundary can be extrapolated to the whole heated region of the plasma column.

The energy balance equations under the above assumptions are:

$$
\begin{aligned}
& \frac{3}{2} \frac{\mathrm{d}}{\mathrm{d} t} n T_{\mathrm{e}}=K_{\nu} I(t)-\frac{3}{2} n \frac{T_{\mathrm{e}}-T_{\mathrm{i}}}{\tau_{\mathrm{e}}}-\beta n^{2} T_{\mathrm{e}}^{1 / 2}, \\
& \frac{3}{2} \frac{\mathrm{d}}{\mathrm{d} t} n T_{\mathrm{i}}=\frac{3}{2} n \frac{T_{\mathrm{e}}-T_{\mathrm{i}}}{\tau_{\mathrm{e}}} .
\end{aligned}
$$

In a plasma confined in a uniform magnetic field (along, say, the $Z$-direction) and in the absence of electric fields, the following relation is obtained from the equation of conservation of momentum [8] (quasistatic pressure assumption):

$$
n\left(T_{\mathrm{e}}+T_{\mathrm{i}}\right)+\frac{B^{2}}{8 \pi}=\frac{B_{0}^{2}}{8 \pi}
$$


By assuming non-diffusivity of the magnetic field [3] we also have:

$$
\frac{n}{B}=\frac{n_{0}}{B_{0}}
$$

In the above equations $n, T_{e}, T_{i}, B$ and $B_{0}$, are respectively the actual ion (electron) density, electron and ion temperatures (in electron-volts) and the internal and external magnetic fields; $n_{0}$ represents the electron and ion densities at negligible temperatures. $K_{\nu}$ and $\tau_{\mathrm{e}}$ are the absorption coefficient of the laser light and the electron-ion equilibration time, given by:

$$
\begin{aligned}
& K_{\nu}=\alpha n^{2} / T_{\mathrm{e}}^{3 / 2}, \\
& \tau_{\mathrm{e}}=\gamma \frac{T_{\mathrm{e}}^{3 / 2}}{n}
\end{aligned}
$$

the numerical coefficients $\alpha, \beta$ and $\gamma$ are explicitly given by:

$$
\begin{aligned}
& \alpha=K \frac{8 \pi e^{6}}{c \nu^{2}\left(2 \pi m_{\mathrm{e}}\right)^{3 / 2}}, \\
& \beta=\frac{32 \pi g(2 \pi)^{1 / 2} e^{6}}{3^{3 / 2} m_{\mathrm{e}}^{3 / 2} c^{3} h}, \\
& \gamma^{-1}=8(2 \pi)^{1 / 2} e^{4} m_{\mathrm{e}}^{1 / 2} \ln \Lambda / 3 m_{\mathrm{i}} .
\end{aligned}
$$

In eq. (7) $K$ is a constant of order $10, m_{\mathrm{e}}$ and $m_{\mathrm{i}}$ are the electron and ion masses, $h$ is the Planck's constant, $c$ is the speed of light, $e$ is the electron charge, $\nu$ is the laser frequency, $g$ is a quantum mechanical correction factor [7] and finally $\ln \Lambda$ is the Coulomb logarithm.

In order to simplify the notation in the above equations, we solve eq. (4) for $B$ and substitute it in (3) to obtain

$$
n T_{\mathrm{e}}+n T_{\mathrm{i}}+\frac{B_{0}^{2}}{8 \pi}\left[\left(n / n_{0}\right)^{2}-1\right]=0
$$

Now, substituting the expressions for $K_{\nu}$ and $\tau_{\mathrm{c}}$, in eqs. (1) and (2), and defining the new variables

$$
\left.\begin{array}{c}
Z_{1} \equiv n T_{\mathrm{e}} / n_{0} \\
Z_{2} \equiv n T_{\mathrm{i}} / n_{0} \\
Z_{3} \equiv(n / n)_{0}^{2} \\
U(t) \equiv I(t)
\end{array}\right\}
$$

the equations describing the laser heating of a plasma are then reduced to

$$
\begin{aligned}
& \frac{\mathrm{d}}{\mathrm{d} t} Z_{1}=\frac{A_{1} Z_{3}^{7 / 4} U(t)}{Z_{1}^{3 / 2}}-\frac{A_{2} Z_{3}^{5 / 4}\left(Z_{1}-Z_{2}\right)}{Z_{1}^{3 / 2}}-A_{3} Z_{3}^{3 / 4} Z_{1}^{1 / 2} \\
& \frac{\mathrm{d}}{\mathrm{d} t} Z_{2}=\frac{A_{2} Z_{3}^{5 / 4}\left(Z_{1}-Z_{2}\right)}{Z_{1}^{3 / 2}}
\end{aligned}
$$

where

$$
Z_{3}=1-A_{4}\left(Z_{1}+Z_{2}\right)
$$


and the coefficients $A_{1}, A_{2}, A_{3}$ and $A_{4}$ are defined as:

$$
A_{1} \equiv \frac{2}{3} \alpha n_{0}, \quad A_{2} \equiv \frac{n_{0}}{\gamma}, \quad A_{3} \equiv \frac{2}{3} \beta n_{0}, \quad A_{4} \equiv \frac{8 \pi n_{0}}{B_{0}^{2}} ;
$$

the system of eqs. (12)-(14) represent our mathematical model of the laser-plasma interaction. The variables $Z_{1}$ and $Z_{2}$ are referred to as state variables and $U(t)$ as the control variable.

In order to illustrate the application of optimal control, in this work we will consider the heating of a neutral plasma composed by ordinary hydrogen ions by a $\mathrm{CO}_{2}$ laser beam of maximum intensity $I_{0}=2.25 \times 10^{30} \mathrm{eV} / \mathrm{cm}^{2} \mathrm{~s}$, where the density $n_{0}$ is $2 \times 10^{17} / \mathrm{cm}^{3}$. Under these circumstances, the numerical values of the coefficients in (15) are

$$
A_{1}=2.667 \times 10^{-17} \mathrm{eV}^{3 / 2} \mathrm{~cm}^{2}, \quad A_{2}=6.349 \times 10^{9} \mathrm{eV}^{3 / 2} / \mathrm{s}, \quad A_{3}=1.404 \times 10^{4} \mathrm{eV} / \mathrm{s}
$$

The value of the coefficient $A_{4}$ (in units $\mathrm{eV}^{-1}$ ) will depend on the magnitude of the external magnetic field used.

Since our problem is concerned with the heating of the plasma ions from an initial state, which is assumed to be known, to a final state which corresponds to the desired final ion temperature, it is of interest to know the analytical form of what is called the "Target Curve", i.e. the set of states in the phase plane $Z_{1}-Z_{2}$ that have the same final ions temperature. By using the definition of state variables in eq. (11), the set of states corresponding to a fixed final ion temperature, $T_{\text {if }}$, is obtained from

$$
Z_{3}^{-1 / 2} Z_{2}=T_{\text {if }}
$$

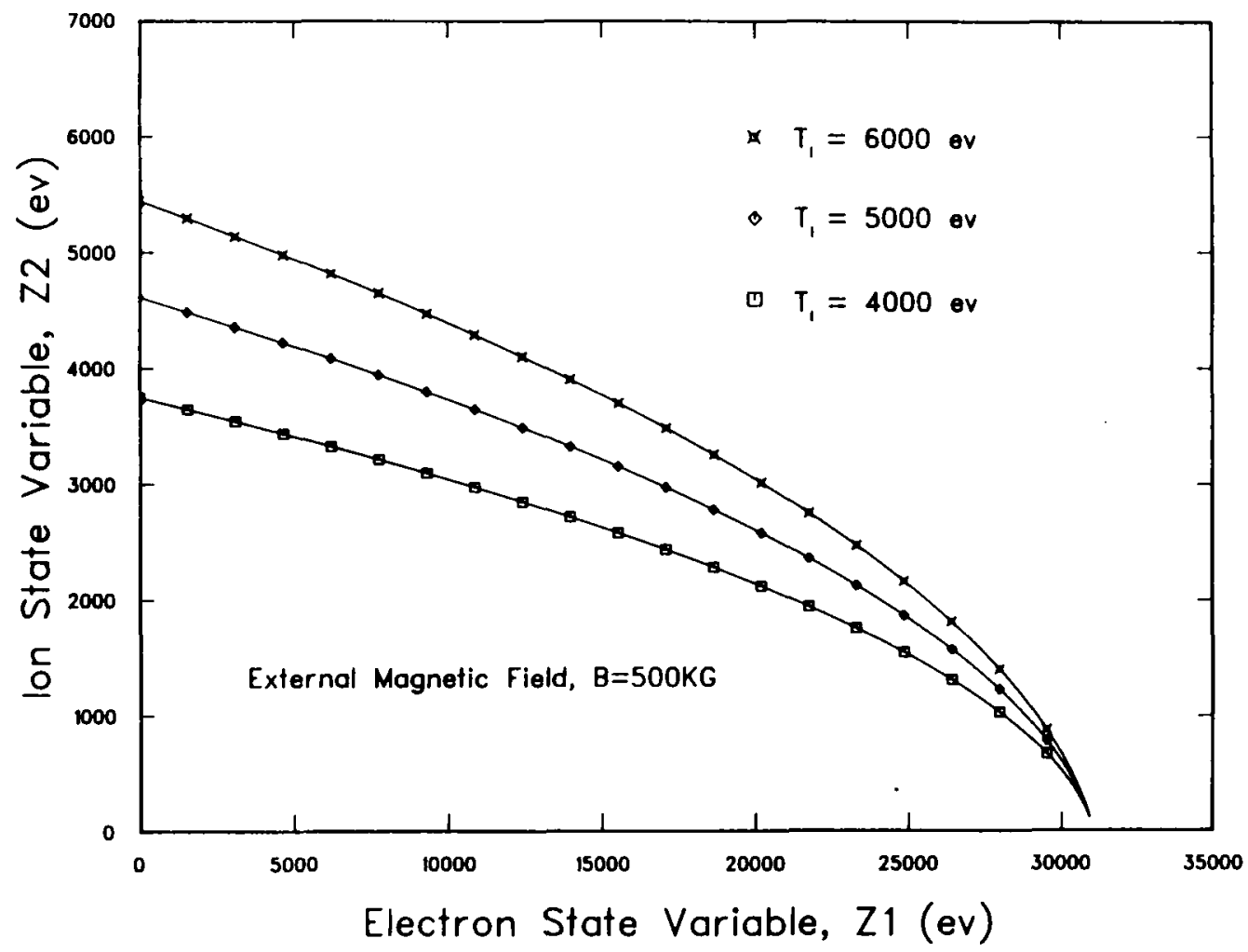

Fig. 1. Target curves for several final ion temperatures when the external magnetic field is $500 \mathrm{kG}$. 


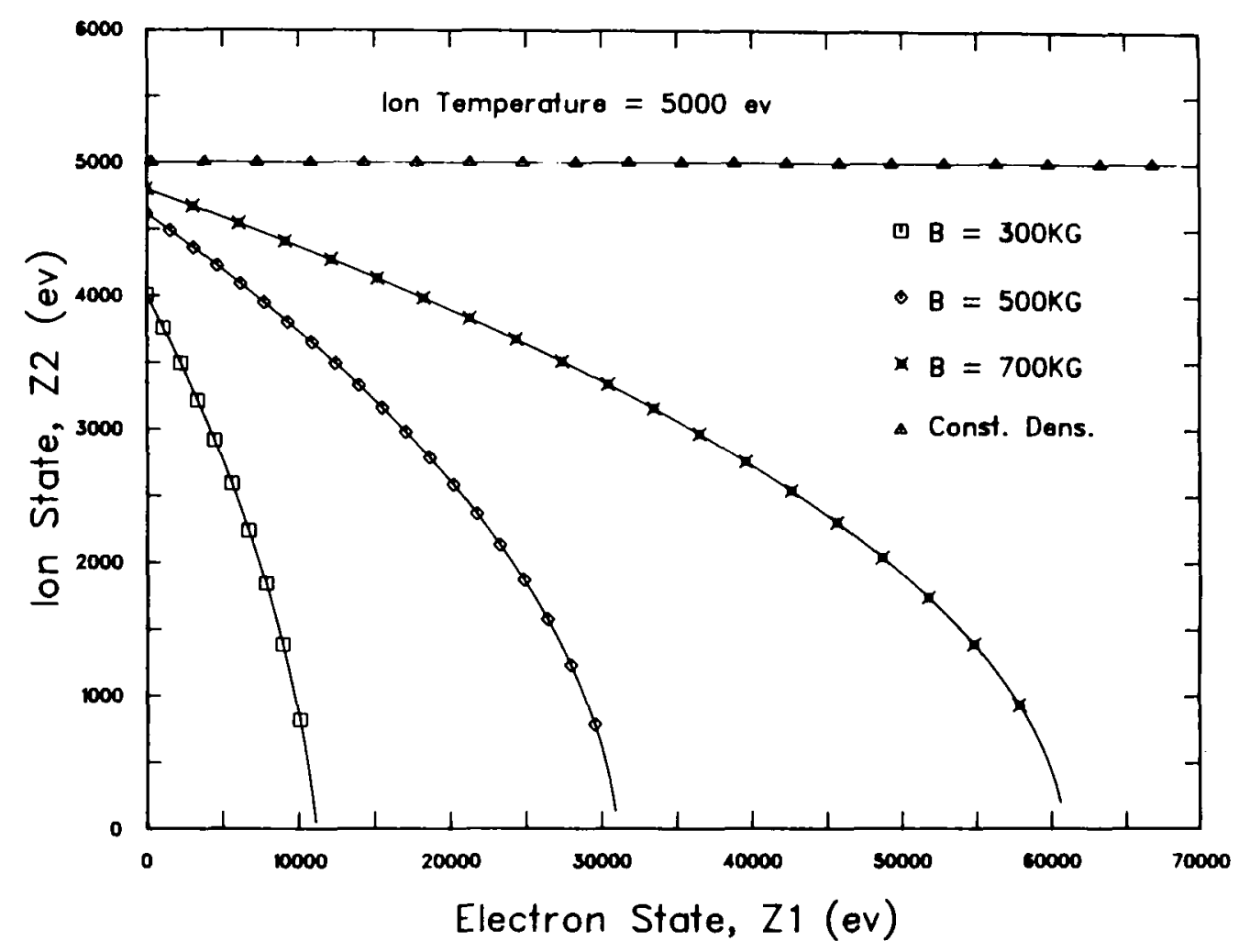

Fig. 2. Target curves corresponding to a final ion temperature of $5 \mathrm{keV}$, for several values of the external magnetic field.

Substituting the expression of $Z_{3}$ from eq. (14) in the above equation, one obtains:

$$
Z_{1}=\left(\frac{1}{A_{4}}+\frac{A_{4} T_{\mathrm{if}}^{2}}{4}\right)-\frac{1}{A_{4} T_{\mathrm{if}}^{2}}\left[Z_{2}+\frac{A_{4} T_{\mathrm{if}}^{2}}{2}\right]^{2}
$$

this is the equation of the target curve in the phase plane. It represents a parabola whose shape depends on the magnitude of the external magnetic field, as well as on the final ion temperature. In the special case of constant density, $Z_{3}=1$, i.e. $A_{4}=0$, the target curve degenerates into a straight line parallel to the $Z_{1}$-axis.

In fig. 1 are shown target curves corresponding to different final ion temperature for a fixed value of the external magnetic field; and fig. 2 shows the behavior of the target curves for a fixed final ion temperature for several values of the external magnetic field.

\section{Optimal control formulation}

\subsection{Hamilton's equations}

The optimal control problem can be formulated as follows [2]:

From all the control values that satisfy $U_{\min } \leq U(t) \leq U_{\max }$, where $U_{\min }=0$ and $U_{\max }=I_{0}$, find the control strategy $U^{\mathrm{OP}}(t)$ which minimize:

$$
J=\int_{0}^{T}\left[C_{1}+C_{2} U(t)\right] \mathrm{d} t, \quad C_{1} \geqslant 0, \quad C_{2} \geqslant 0,
$$


where $C_{1}$ and $C_{2}$ are weighting parameters, and brings the system, through the eqs. (12)-(14), from a specified initial state

$$
Z_{1}(0)=Z_{10}, \quad Z_{2}(0)=Z_{20},
$$

to a final state with specified ion temperature $T_{\mathrm{if}}$, represented by the terminal condition

$$
\left.M\left(Z_{1}, Z_{2}\right)\right|_{T}=0
$$

where

$$
M\left(Z_{1}, Z_{2}\right) \equiv T_{\text {if }}-Z_{3}^{-1 / 2} Z_{2}
$$

The heating time $T$ is not specified.

The necessary conditions for optimality require the use of the Hamiltonian $H$ :

$$
H=C_{1}+C_{2} U+\lambda_{1}\left[\frac{A_{1} Z_{3}^{7 / 4} U}{Z_{1}^{3 / 2}}-A_{2} \frac{Z_{3}^{5 / 4}\left(Z_{1}-Z_{2}\right)}{Z_{1}^{3 / 2}}-A_{3} Z_{3}^{3 / 4} Z_{1}^{1 / 2}\right]+\lambda_{2}\left[\frac{A_{2} Z_{3}^{5 / 4}\left(Z_{1}-Z_{2}\right)}{Z_{1}^{3 / 2}}\right],
$$

where $Z_{1}, Z_{2}$ are the state variables and $\lambda_{1}, \lambda_{2}$ are the adjoint variables. The optimal solution should satisfy the Hamilton equations [2]:

$$
\begin{aligned}
& \frac{\mathrm{d}}{\mathrm{d} t} Z_{1}= \frac{A_{1} Z_{3}^{7 / 4} U(t)}{Z_{1}^{3 / 2}}-A_{2} \frac{Z_{3}^{5 / 4}\left(Z_{1}-Z_{2}\right)}{Z_{1}^{3 / 2}}-A_{3} Z_{3}^{3 / 4} Z_{1}^{1 / 2} \\
& \frac{\mathrm{d}}{\mathrm{d} t} Z_{2}= A_{2} \frac{Z_{3}^{5 / 4}\left(Z_{1}-Z_{2}\right)}{Z_{1}^{3 / 2}} \\
& \frac{\mathrm{d}}{\mathrm{d} t} \lambda_{1}= \frac{\lambda_{1}}{4 Z_{3}^{1 / 4} Z_{1}^{5 / 2}}\left[6 A_{1} Z_{3}^{2} U+7 A_{1} A_{4} Z_{3} Z_{1} U-2 A_{2} Z_{3}^{3 / 2} Z_{1}+6 A_{2} Z_{3}^{3 / 2} Z_{2}+2 A_{3} Z_{3} Z_{1}^{2}\right. \\
&\left.-5 A_{2} A_{4} Z_{3}^{1 / 2}\left(Z_{1}-Z_{2}\right) Z_{1}-3 A_{3} A_{4} Z_{1}^{3}\right] \\
&+\frac{\lambda_{2} Z_{3}^{1 / 4}}{4 Z_{1}^{5 / 2}}\left[2 A_{2} Z_{3}\left(Z_{1}-3 Z_{2}\right)+5 A_{2} A_{4}\left(Z_{1}-Z_{2}\right) Z_{1}\right] \\
& \frac{\lambda_{1} Z_{3}^{1 / 4}}{4 Z_{1}^{3 / 2}}\left[7 A_{1} A_{4} Z_{3}^{1 / 2} U-4 A_{2} Z_{3}-5 A_{2} A_{4}\left(Z_{1}-Z_{2}\right)\right]+\frac{\lambda_{2} A_{2} Z_{3}^{1 / 4}}{4 Z_{1}^{3 / 2}}\left[4 Z_{3}+5 A_{4}\left(Z_{1}-Z_{2}\right)\right] \\
& \frac{\mathrm{d}}{\mathrm{d} t} \lambda_{2}
\end{aligned}
$$

the optimal control is given by

$$
U^{\mathrm{op}}(t)= \begin{cases}U_{\max } & \text { if } C_{2}+\frac{A_{1} \lambda_{1} Z_{3}^{7 / 4}}{Z_{1}^{3 / 2}}<0 \\ 0 & \text { if } C_{2}+\frac{A_{1} \lambda_{1} Z_{3}^{7 / 4}}{Z_{1}^{3 / 2}}>0 \\ U_{s}(t) & \text { if } C_{2}+\frac{A_{1} \lambda_{1} Z_{3}^{7 / 4}}{Z_{1}^{3 / 2}}=0\end{cases}
$$


where $U_{\mathrm{s}}$ is called the singular control $[9,10]$. The transversality and final conditions [2] lead to:

$$
\begin{aligned}
& \left.H(\bar{Z}, \bar{\lambda})\right|_{T}=0, \\
& \bar{\lambda}(T)=\left.\xi\left(\frac{\partial M}{\partial \bar{Z}}\right)^{\dagger}\right|_{T}, \\
& \left.M\left(Z_{1}, Z_{2}\right)\right|_{T}=0,
\end{aligned}
$$

where $\xi$ is a constant Lagrange multiplier to be determined.

Since the Hamiltonian $H$, is not explicit function of time, we also have:

$$
H(\bar{Z}, \bar{\lambda})=0 \text { for } 0 \leq t \leq T .
$$

In order to isolate those candidates that satisfy the above conditions, it is necessary to analyze the existence and optimality of singular arcs [10].

\subsection{Singular subarcs candidates}

Following Gabasov and Kirillova [9], we divide the Hamiltonian in two parts:

$$
H=H_{0}(\bar{Z}, \bar{\lambda})+H_{1}(\bar{Z}, \bar{\lambda}) U,
$$

where,

$$
H_{0}=C_{1}+\frac{\lambda_{1} Z_{3}^{3 / 4}}{Z_{1}^{3 / 2}}\left[A_{2} Z_{3}^{1 / 2}\left(Z_{2}-Z_{1}\right)-A_{3} Z_{1}^{2}\right]+\frac{A_{2} \lambda_{2} Z_{3}^{5 / 4}}{Z_{1}^{3 / 2}}\left(Z_{1}-Z_{2}\right),
$$

and

$$
H_{1}=C_{2}+A_{1} \lambda_{1} Z_{3}^{7 / 4} / Z_{1}^{3 / 2} .
$$

The singular subarc exists if the switching function $H_{1}$ is equal to zero during an interval of time of positive length. If the phase space trajectory defined by eqs. (24)-(27) satisfies this condition, it is then required that higher order total time derivatives of $H_{1}$, should also vanish. It can be shown that the total time derivatives of $H_{1}$ can be written as:

$$
\frac{\mathrm{d}^{m}}{\mathrm{~d} t^{m}} H_{1}=\alpha_{m}(\bar{Z}, \bar{\lambda})+\beta_{m}(\bar{Z}, \bar{\lambda}) U, \quad m=0,1, \ldots, 2 q,
$$

this process of derivation continues until for some integer number " $q$ ", the coefficient $\beta_{2 q}$ is not identically zero. Then the singular control problem is said to be of order " $q$ " [10].

In this problem, $q=1$ and the coefficients $\alpha_{m}$ and $\beta_{m}$ are given by:

$$
\begin{aligned}
\alpha_{0}= & C_{2}+A_{1} \lambda_{1} Z_{3}^{7 / 4} / Z_{1}^{3 / 2}, \\
\beta_{0} \equiv & 0, \\
\alpha_{1}= & \frac{A_{1} Z_{3}^{3 / 2}}{4 Z_{1}^{4}}\left\{\lambda_{1}\left[4 A_{2} Z_{3}^{3 / 2} Z_{1}+8 A_{3} Z_{3} Z_{1}^{2}-5 A_{2} A_{4} Z_{3}^{1 / 2}\left(Z_{1}-Z_{2}\right) Z_{1}-10 A_{3} A_{4} Z_{1}^{3}\right]\right. \\
& \left.+\lambda_{2}\left[2 A_{2} Z_{3}^{3 / 2}\left(Z_{1}-3 Z_{2}\right)+5 A_{2} A_{4} Z_{3}^{1 / 2}\left(Z_{1}-Z_{2}\right) Z_{1}\right]\right\},
\end{aligned}
$$




$$
\begin{aligned}
\beta_{1} \equiv & 0, \\
\alpha_{2}= & -\frac{4 \alpha_{1}}{Z_{1}^{5 / 2}}\left\{-A_{2} Z_{3}^{5 / 4} Z_{1}+A_{2} Z_{3}^{5 / 4} Z_{2}-A_{3} Z_{3}^{3 / 4} Z_{1}^{2}\right\} \\
& +\lambda_{1} \frac{A_{1} Z_{3}^{5 / 4}}{16 Z_{1}^{11 / 2}}\left\{-32 A_{2}^{2} Z_{3}^{3} Z_{1}+64 A_{2}^{2} Z_{3}^{3} Z_{2}-88 A_{2} A_{3} Z_{3}^{5 / 2} Z_{1}^{2}+20 A_{2}^{2} A_{4} Z_{3}^{2}\left(Z_{1}-Z_{2}\right)^{2}\right. \\
& +112 A_{2} A_{3} Z_{3}^{5 / 2} Z_{1} Z_{2}-48 A_{3}^{2} Z_{3}^{2} Z_{1}^{3}+166 A_{2} A_{3} A_{4} Z_{3}^{3 / 2} Z_{1}^{3}-150 A_{2} A_{3} A_{4} Z_{3}^{3 / 2} Z_{2} Z_{1}^{2} \\
& \left.+156 A_{3}^{2} A_{4} Z_{3} Z_{1}^{4}+25 A_{2} A_{3} A_{4}^{2} Z_{3}^{1 / 2}\left(Z_{1}-Z_{2}\right) Z_{1}^{3}-30 A_{3}^{2} A_{4}^{2} Z_{1}^{5}\right\} \\
& +\lambda_{2} \frac{A_{1} Z_{3}^{7 / 4}}{8 Z_{1}^{11 / 2}}\left\{-8 A_{2}^{2} Z_{3}^{5 / 2}\left(Z_{1}-Z_{2}\right)-10 A_{2}^{2} A_{4} Z_{3}^{3 / 2}\left(Z_{1}-Z_{2}\right)^{2}+2 A_{2} A_{3} A_{4} Z_{3} Z_{1}^{3}\right. \\
& \left.-16 A_{2} A_{3} A_{4} Z_{3} Z_{2} Z_{1}^{2}+4 A_{2} A_{3} Z_{3}^{2} Z_{1}^{2}-5 A_{2} A_{3} A_{4}^{2}\left(Z_{1}-Z_{2}\right) Z_{1}^{3}-24 A_{2} A_{3} Z_{3}^{2} Z_{2} Z_{1}\right\} \\
\beta_{2}= & -\frac{4 \alpha_{1} A_{1} Z_{3}^{7 / 4}}{Z_{1}^{5 / 2}}+\frac{A_{1}^{2} Z_{3}^{9 / 4}}{8 Z_{1}^{11 / 2}}\left\{\lambda _ { 1 } \left[20 A_{2} Z_{3}^{5 / 2}-38 A_{2} A_{4} Z_{3}^{3 / 2} Z_{1}+4 A_{2} A_{4} Z_{3}^{3 / 2} Z_{2}+56 A_{3} Z_{3}^{2} Z_{1}\right.\right. \\
& \left.-102 A_{3} A_{4} Z_{3} Z_{1}^{2}-5 A_{3} A_{4}^{2} Z_{1}^{3}+20 A_{2} A_{4}^{2} Z_{3}^{1 / 2}\left(Z_{1}-Z_{2}\right) Z_{1}\right] \\
& \left.+\lambda_{2}\left[4 A_{2} Z_{3}^{5 / 2}+8 A_{2} A_{4} Z_{3}^{3 / 2} Z_{1}+26 A_{2} A_{4} Z_{3}^{3 / 2} Z_{2}-20 A_{2} A_{4}^{2} Z_{3}^{1 / 2}\left(Z_{1}-Z_{2}\right) Z_{1}\right]\right\}
\end{aligned}
$$

Since $\beta_{2}$ does not vanish identically we have a singular arc of order 1 .

The singular subarc is specified by:

$$
\begin{aligned}
0= & C_{2}+A_{1} \lambda_{1} Z_{3}^{7 / 4} / Z_{1}^{3 / 2}, \\
0= & \lambda_{1}\left[4 A_{2} Z_{3}^{3 / 2} Z_{1}+8 A_{3} Z_{3} Z_{1}^{2}-5 A_{2} A_{4} Z_{3}^{1 / 2}\left(Z_{1}-Z_{2}\right) Z_{1}-10 A_{3} A_{4} Z_{1}^{3}\right] \\
& +\lambda_{2}\left[2 A_{2} Z_{3}^{3 / 2}\left(Z_{1}-3 Z_{2}\right)+5 A_{2} A_{4} Z_{3}^{1 / 2}\left(Z_{1}-Z_{2}\right) Z_{1}\right] .
\end{aligned}
$$

In order to obtain the singular control as a function of $Z_{1}$ and $Z_{2}$ we solve first the above equations for $\lambda_{1}$ and $\lambda_{2}$, to obtain:

$$
\begin{aligned}
& \lambda_{1}=-C_{2} Z_{1}^{3 / 2} / A_{1} Z_{3}^{7 / 4} \\
& \lambda_{2}=\left(\frac{C_{2} Z_{1}^{5 / 2}}{A_{1} A_{2} Z_{3}^{9 / 4}}\right) \frac{4 A_{2} Z_{3}^{3 / 2}+8 A_{3} Z_{1} Z_{3}-5 A_{2} A_{4} Z_{3}^{1 / 2}\left(Z_{1}-Z_{2}\right)-10 A_{3} A_{4} Z_{1}^{2}}{2 Z_{3}\left(Z_{1}-3 Z_{2}\right)+5 A_{4} Z_{1}\left(Z_{1}-Z_{2}\right)} .
\end{aligned}
$$

Substituting these expressions in the equations for $\alpha_{2}$ and $\beta_{2}$ and requiring the second order time derivative of $H_{1}$ to vanish, we obtain for the singular control:

$$
U_{s}=\frac{N\left(Z_{1}, Z_{2}\right)}{D\left(Z_{1}, Z_{2}\right)}
$$

where $N\left(Z_{1}, Z_{2}\right)$ and $D\left(Z_{1}, Z_{2}\right)$ are given by:

$$
\begin{aligned}
N\left(Z_{1}, Z_{2}\right)= & 24 A_{2}^{2} Z_{3}^{4}\left(Z_{1}-Z_{2}\right) Z_{2}+A_{2} A_{3} Z_{3}^{7 / 2}\left(67 Z_{2} Z_{1}-5 Z_{1}^{2}-42 Z_{2}^{2}\right) Z_{1} \\
& +A_{3}^{2} Z_{3}^{3}\left(42 Z_{2}-10 Z_{1}\right) Z_{1}^{3}-15 A_{2}^{2} A_{4} Z_{3}^{3}\left[Z_{1}^{3}-Z_{2} Z_{1}^{2}-Z_{2}^{2} Z_{1}+Z_{2}^{3}\right] / 2 \\
& +A_{2} A_{3} A_{4} Z_{3}^{5 / 2}\left[-21 Z_{1}^{2}-232 Z_{2} Z_{1}+185 Z_{2}^{2}\right] Z_{1}^{2} / 4+A_{2} A_{3} A_{4}^{2} Z_{3}^{3 / 2} \\
& \times\left[185 Z_{1}^{5}-400 Z_{2} Z_{1}^{4}+215 Z_{2}^{2} Z_{1}^{3}\right] / 4+A_{3}^{2} A_{4} Z_{3}^{2}\left[15 Z_{1}-115 Z_{2}\right] Z_{1}^{4} / 2 \\
& +A_{3}^{2} A_{4}^{2} Z_{3}\left[105 Z_{1}-125 Z_{2}\right] Z_{1}^{5} / 2+75 A_{2} A_{3} A_{4}^{3} Z_{3}^{1 / 2}\left[Z_{1}-Z_{2}\right]^{2} Z_{1}^{4} / 16 \\
& -125 A_{3}^{2} A_{4}^{3}\left(Z_{1}-Z_{2}\right) Z_{1}^{6} / 8,
\end{aligned}
$$




$$
\begin{aligned}
D\left(Z_{1}, Z_{2}\right)= & A_{1} Z_{3}\left\{A_{2} Z_{3}^{7 / 2}\left(15 Z_{2}-3 Z_{1}\right)+A_{3} Z_{3}^{3}\left(42 Z_{2}-10 Z_{1}\right) Z_{1}\right. \\
& +A_{2} A_{4} Z_{3}^{5 / 2}\left(6 Z_{2}^{2}-3 Z_{1} Z_{2}-3 Z_{1}^{2}\right) / 2 \\
& -A_{3} A_{4} Z_{3}^{2}\left(13 Z_{1}+31 Z_{2}\right) Z_{1}^{2} / 2+15 A_{2} A_{4}^{2} Z_{3}^{3 / 2}\left(Z_{1}-Z_{2}\right)^{2} Z_{1} / 4 \\
& \left.+A_{3} A_{4}^{2} Z_{3}\left(35 Z_{1}-80 Z_{2}\right) Z_{1}^{3}+225 A_{3} A_{4}^{3}\left(Z_{1}-Z_{2}\right) Z_{1}^{4} / 8\right\}
\end{aligned}
$$

where we should keep in mind that $Z_{3}=1-A_{4}\left(Z_{1}+Z_{2}\right)$.

The above expression for the singular control is independent of $C_{1}$ and $C_{2}$, and it reduces to the corresponding equation for the singular control in the particular case of constant plasma density, i.e. when $A_{4}=0$.

\subsection{Optimality of singular subarcs}

Singular arcs are optimal candidates if they satisfy the Generalized Legendre-Clebsh condition (GLC) [11]. In the case of singular control problems of order 1 , this condition is

$$
\text { i.e., } \left.\quad \begin{array}{l}
\frac{\partial}{\partial U} \frac{\mathrm{d}^{2} H_{1}}{\mathrm{~d} t^{2}} \leq 0 \\
\beta_{2}(\bar{Z}, \bar{\lambda}) \leq 0 .
\end{array}\right\}
$$

In order to determine if the singular arc satisfies this condition, we will use the fact that the Hamiltonian is a constant of motion along those trajectories that satisfy the necessary conditions for optimality. Thus, using eqs. (23), (30) and (38), we obtain an alternative expression for $\lambda_{2}$ along singular arcs, which is

$$
\lambda_{2}=\lambda_{1}\left[1+\frac{A_{3} Z_{1}^{2}}{A_{2} Z_{3}^{1 / 2}\left(Z_{1}-Z_{2}\right)}+\frac{A_{1} C_{1} Z_{3}^{1 / 2}}{A_{2} C_{2}\left(Z_{1}-Z_{2}\right)}\right]
$$

Substituting the above expression together with (37) and (38) in the equation for $\beta_{2}$ in (35f), the GLC condition becomes

$$
\begin{gathered}
C_{2}\left[A_{2} Z_{3}^{3 / 2}\left(3 Z_{3}-\frac{15}{4} A_{4}\left(Z_{1}-Z_{2}\right)\right)+A_{3} Z_{1}\left(7 Z_{3}^{2}-\frac{51}{4} A_{4} Z_{3} Z_{1}-\frac{25}{8} A_{4}^{2} Z_{1}^{2}\right)\right] \\
+\frac{C_{2} A_{3} Z_{3} Z_{1}^{2}+C_{1} A_{1} Z_{3}^{2}}{2\left(Z_{1}-Z_{2}\right)}\left(Z_{3}+2 A_{4} Z_{1}+\frac{13}{2} A_{4} Z_{2}\right)-\frac{5}{2} A_{1} A_{4}^{2} Z_{3} Z_{1} C_{1} \geq 0
\end{gathered}
$$

In the region of the phase plane where the ion heating takes place $\left(Z_{1}>Z_{2}\right)$, the GLC condition will be satisfied if the parameter $A_{4}$ is small, or if the electron and ion states values are not very large. In our subsequent discussions we will assume that the magnetic field is strong enough, i.e. $A_{4}$ is small, such that the GLC condition is always satisfied.

\subsection{Equation of the singular arcs}

The algebraic equation specifying the singular subarcs is obtained by equating the expressions for $\lambda_{2}$ given in (39) and (44). We obtain:

$$
\begin{aligned}
0= & 6 A_{2} C_{2} Z_{3}^{3 / 2} Z_{1}^{2}-12 A_{2} C_{2} Z_{3}^{3 / 2} Z_{2} Z_{1}+6 A_{2} C_{2} Z_{3}^{3 / 2} Z_{2}^{2}+10 A_{3} C_{2} Z_{3} Z_{1}^{3}-14 A_{3} C_{2} Z_{3} Z_{2} Z_{1}^{2} \\
& +2 A_{1} C_{1} Z_{3}^{2} Z_{1}-6 A_{1} C_{1} Z_{3}^{2} Z_{2}+5 A_{1} A_{4} C_{1} Z_{3} Z_{1}\left(Z_{1}-Z_{2}\right)-5 A_{3} A_{4} C_{2}\left(Z_{1}-Z_{2}\right) Z_{1}^{3}
\end{aligned}
$$

where $Z_{3}=1-A_{4}\left(Z_{1}+Z_{2}\right)$. The above expression for the singular subarc depends on the relative 
magnitude of $C_{1} / C_{2}$ and on the magnitude of the magnetic field, through the parameter $A_{4}$. Note that this expression reduces to that corresponding to the constant density case $[12,13]$ when $A_{4}=0$ (i.e. $Z_{3}=1$ ).

From the general equation for the singular arc in (46) the corresponding equations for the limiting cases of minimum time subarcs $\left(C_{1}=0, C_{2} \neq 0\right)$ can be easily obtained as:

$$
\begin{aligned}
& 0=2 Z_{3}\left(Z_{1}-3 Z_{2}\right)+5 A_{4} Z_{1}\left(Z_{1}-Z_{2}\right) \quad \text { (minimum time) } \\
& 0=6 A_{2} Z_{3}^{3 / 2}\left(Z_{1}-Z_{2}\right)^{2}+10 A_{3} Z_{3} Z_{1}^{3}-14 A_{3} Z_{3} Z_{2} Z_{1}^{2}-5 A_{3} A_{4}\left(Z_{1}-Z_{2}\right) Z_{1}^{3} \quad \text { (minimum energy). }
\end{aligned}
$$

The eq. (47) corresponding to singular arcs for the case of minimum time trajectories has the physical meaning that, when the laser intensity profile is shaped according to eq. (40) to keep the electron and ion temperatures satisfying (47), the energy transfer rate between electrons and ions represented by the term $A_{2} Z_{3}^{5 / 4}\left(Z_{1}-Z_{2}\right) / Z_{1}^{3 / 2}$, is maximized.

Due to the constraint in the maximum laser intensity available (recall $U_{\min } \leq U(t) \leq U_{\max }$, with $U_{\min }=0$ and $U_{\max }=I_{0}$ ), two different possibilities exist:

(a) It may happen that the singular control along the singular arc, reaches the upper limit; thus singular subarcs will contain an upper bound that depends on the maximum laser intensity, as well as on the ratio $C_{1} / C_{2}$, that will be called "Laser Saturation State".

(b) On the other hand, it may be possible that the singular control increases along the singular arc until a local maximum is reached and then it starts to decrease. This behavior is expected when the density of the plasma decreases to a point in which optimal absorption of the laser energy is obtained derreasing its intensity.

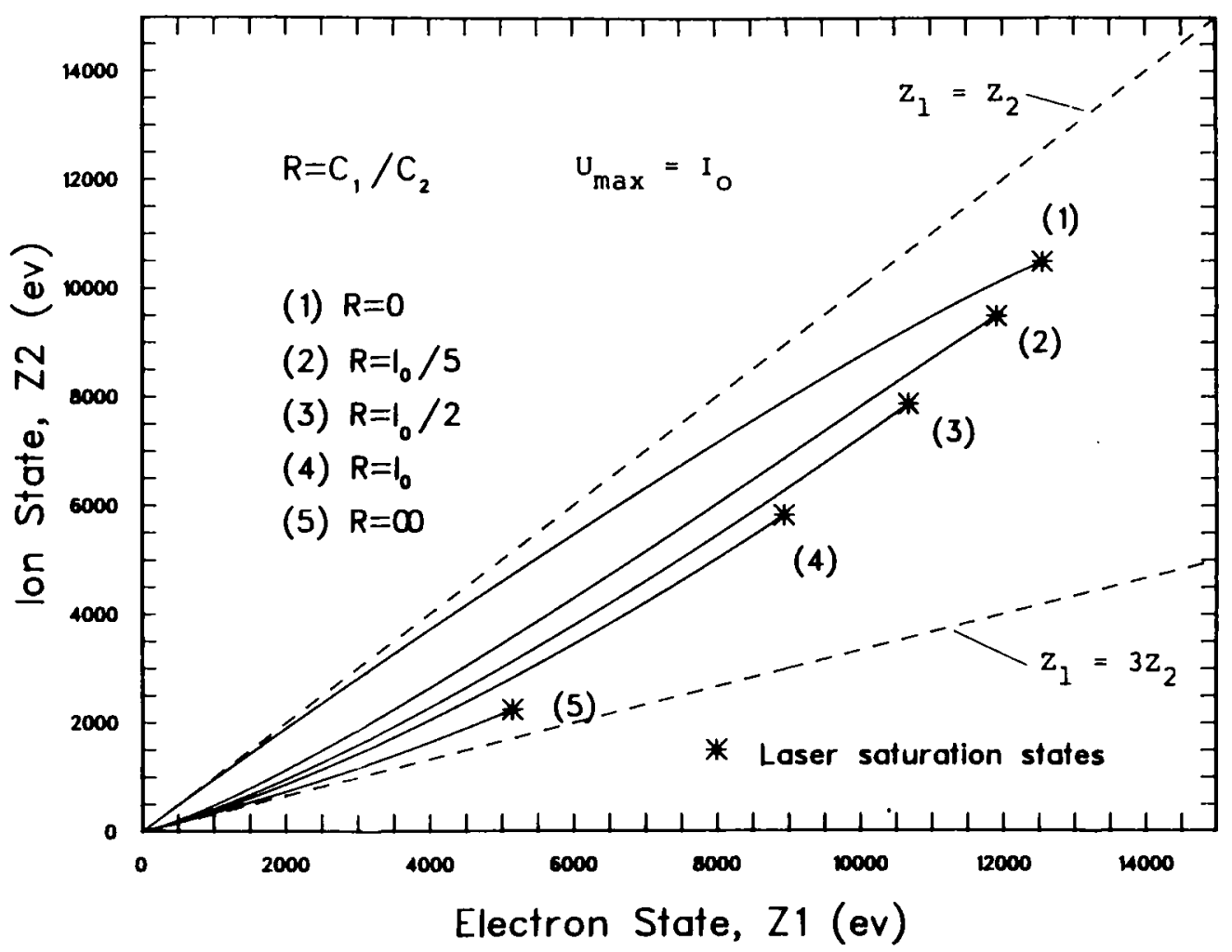

Fig. 3. Behavior of singular subarcs for several ratios of the weighting parameters, when the external magnetic field is $500 \mathrm{kG}$. 


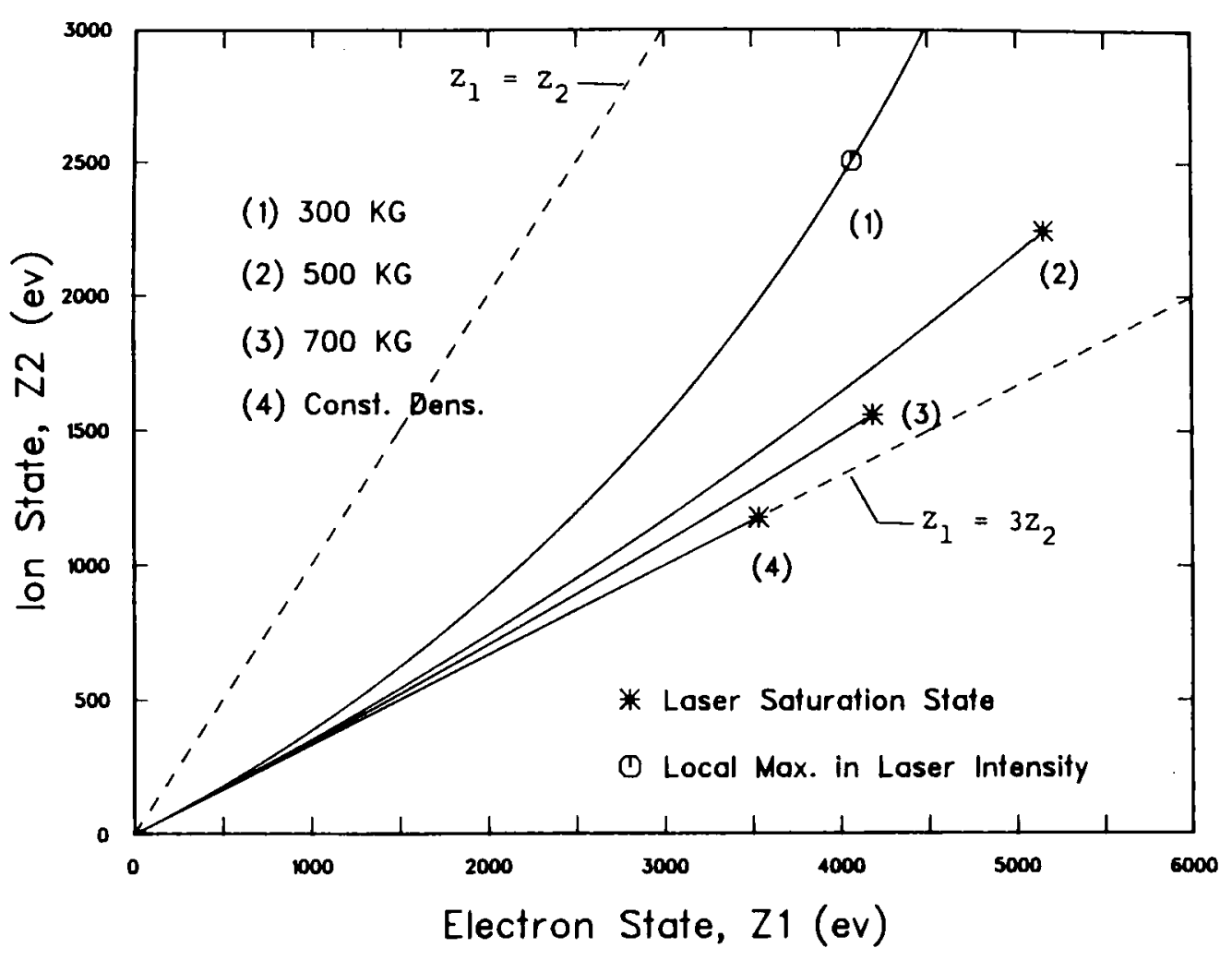

Fig. 4. Behavior of singular arcs corresponding to minimun time trajectories for several values of the external magnetic field.

Fig. 3 shows the behavior of singular arcs for several values of the ratio $C_{1} / C_{2}$, corresponding to an external magnetic field of 500 kiloGauss. The behavior of Minimum Time and Minimum Energy singular arcs for several values of the external magnetic field are shown in figs. 4 and 5. And in fig. 6 the singular arcs corresponding to $C_{1} / C_{2}=I_{0}$ are plotted for several values of the external magnetic field. Figs. 4 and 6 show the appearance of local maximums in the laser intensity when the external magnetic field decreases.

It has to be pointed out, that, Minimum Energy singular arcs $\left(C_{1}=0\right)$, degenerate into the $Z_{1}=Z_{2}$ straight line if no radiation losses are included $\left(A_{3}=0\right)$. This is expected since this trajectory maximize the laser-electrons coupling; we will return to this point later.

\subsection{Terminal and control switching conditions}

Analyzing the transversality and final conditions (29) it is possible to separate the final state and switching points conditions for three different cases [12]:

3.5.1. Minimum time trajectories $C_{1}=1, C_{2}=0$

At the final time $T$, the state and adjoint variables should satisfy the following conditions,

$$
\begin{aligned}
& Z_{1}(T)>T_{\text {if }}\left[1+A_{4}^{2} T_{\text {if }}^{2}\right]^{1 / 2}-A_{4} T_{\text {if }}^{2}, \text { i.e. } Z_{1}(T)>Z_{2}(T) . \\
& Z_{2}(T)=\frac{T_{\text {if }}}{2}\left\{\left[A_{4}^{2} T_{\text {if }}^{2}+4\left(1-A_{4} Z_{1}(T)\right)\right]^{1 / 2}-A_{4} T_{\text {if }}\right\}, \\
& \lambda_{1}(T)=\frac{-A_{4} Z_{1}^{3 / 2} Z_{3}^{-3 / 4} T_{\text {if }}}{2 A_{2} Z_{3}\left(Z_{1}-Z_{2}\right)-A_{3} A_{4} Z_{1}^{2} T_{\text {if }}+A_{1} A_{4} U_{\max } T_{\text {if }} Z_{3}},
\end{aligned}
$$




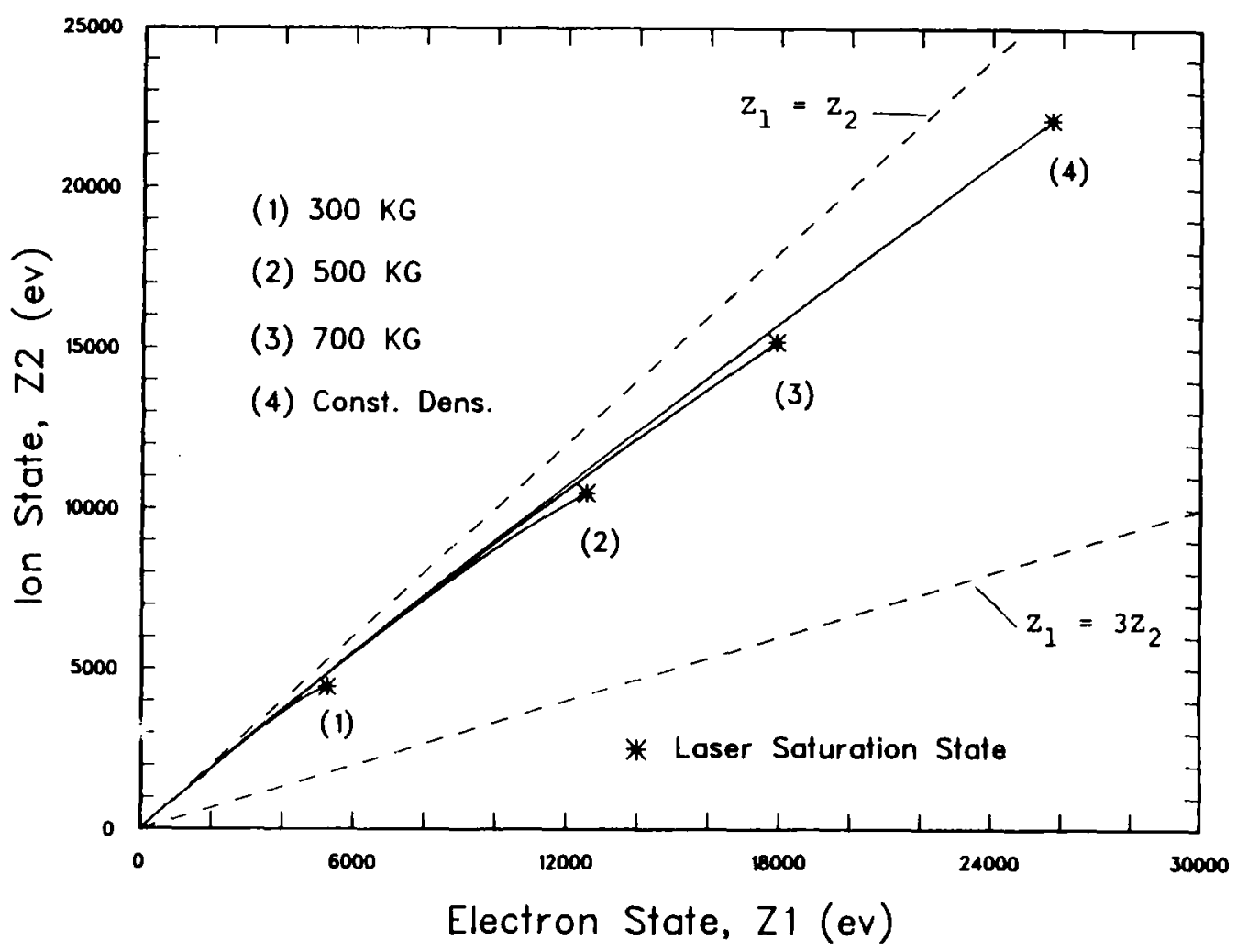

Fig. 5. Behavior of minimum energy singular arcs, for several values of the external magnetic field.

and

$$
\lambda_{2}(T)=\frac{-Z_{1}^{3 / 2} Z_{3}^{-3 / 4}\left[2 Z_{3}^{1 / 2}+A_{4} T_{\mathrm{if}}\right]}{2 A_{2} Z_{3}\left(Z_{1}-Z_{2}\right)-A_{3} A_{4} Z_{1}^{2} T_{\mathrm{if}}+A_{1} A_{4} U_{\max } T_{\mathrm{if}} Z_{3}} .
$$

The optimal control at the final states is always

$$
U^{\mathrm{op}}=U_{\max } \text {. }
$$

If an optimal control switching exists, say at time $t_{\mathrm{s}}$, the following conditions should hold:

$$
\begin{aligned}
& \lambda_{1}\left(t_{\mathrm{s}}\right)=0, \\
& \lambda_{2}\left(t_{\mathrm{s}}\right)=-\left.\frac{Z_{1}^{3 / 2}}{A_{2} Z_{3}^{5 / 4}\left(Z_{1}-Z_{2}\right)}\right|_{t_{\mathrm{s}}} .
\end{aligned}
$$

Together with a control switching of the form:

$$
\begin{array}{ll}
U_{\max } \rightarrow U_{\min } & \text { if } S<0, \\
U_{\min } \rightarrow U_{\max } & \text { if } S>0, \\
U_{\max } \leftrightarrow U_{\mathrm{s}} \\
\text { or } \\
\left.\begin{array}{l}
U_{\operatorname{mi}} \rightarrow U_{\mathrm{s}}
\end{array}\right\} & \text { if } S=0,
\end{array}
$$




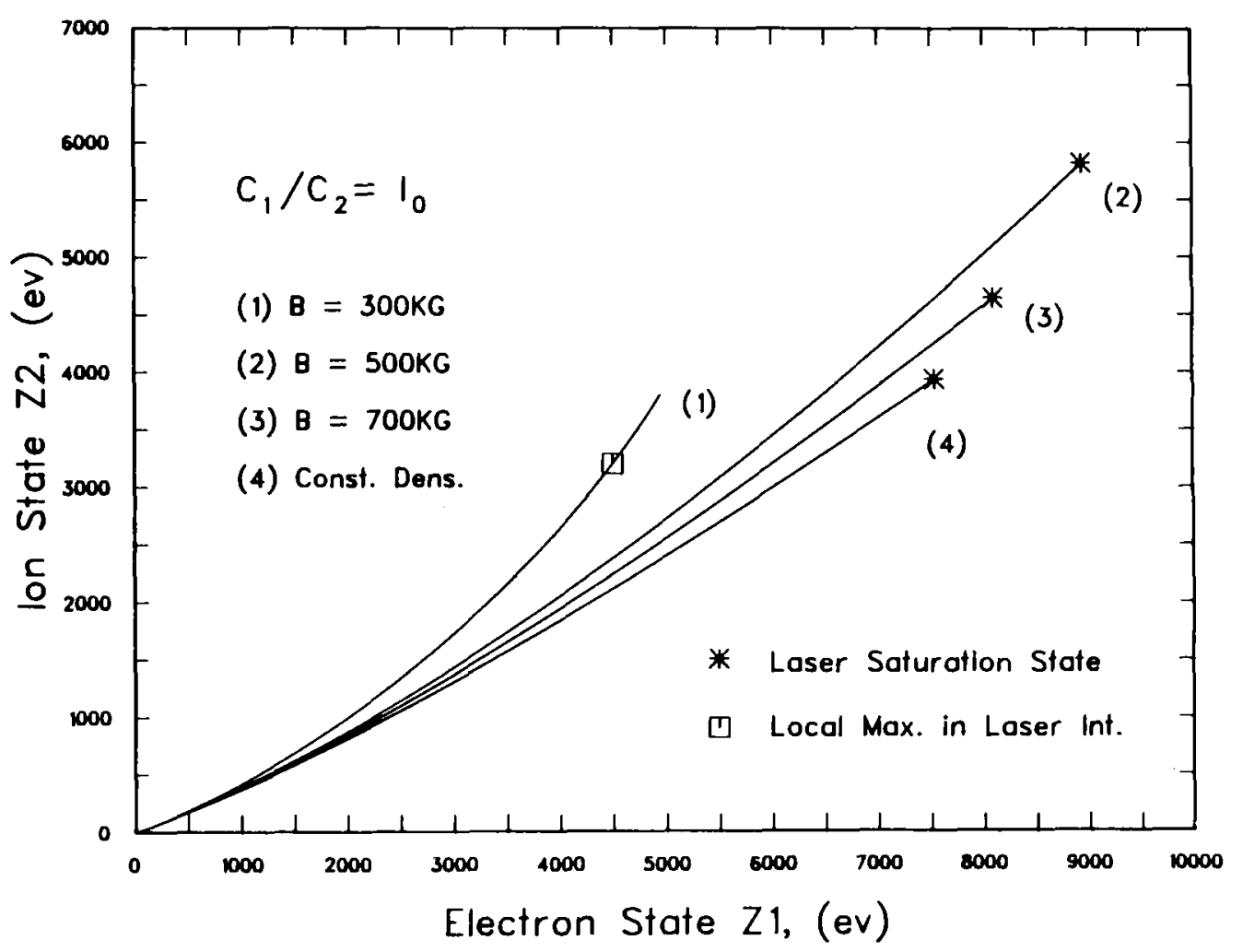

Fig. 6. Behavior of singular arcs corresponding to a ratio $C_{1} / C_{2}=U_{\max }$, for several values of the external magnetic field.

where $S$, the singular function is given by:

$$
S=2 Z_{3}\left(Z_{1}-3 Z_{2}\right)+5 A_{4} Z_{1}\left(Z_{1}-Z_{2}\right) \text {, }
$$

where as always, $Z_{3}=1-A_{4}\left(Z_{1}+Z_{2}\right)$.

3.5.2. Minimum energy trajectories, $C_{1}=0, C_{2}=1$

At the final states minimum energy trajectories should satisfy the following terminal conditions:

$$
\begin{aligned}
& Z_{1}(T)>T_{\mathrm{if}}\left[1+A_{4}^{2} T_{\mathrm{if}}^{2}\right]^{1 / 2}-A_{4} T_{\mathrm{if}}^{2}, \text { i.e., } Z_{1}(T)>Z_{2}(T), \\
& Z_{2}(T)=\frac{T_{\mathrm{if}}}{2}\left\{\left[A_{4}^{2} T_{\mathrm{if}}^{2}+4\left(1-A_{4} Z_{1}(T)\right)\right]^{1 / 2}-A_{4} T_{\mathrm{if}}\right\}
\end{aligned}
$$

For the adjoint variables three cases may occur:

(i) If the final state, $Z_{1}(T)$ and $Z_{2}(T)$, satisfies, $2 A_{2} Z_{3}\left(Z_{1}-Z_{2}\right)<A_{3} A_{4} Z_{1}^{2} T_{\text {if }}$, then:

$$
\lambda_{1}(T)=\frac{-A_{4} Z_{1}^{3 / 2} Z_{3}^{-3 / 4} U_{\max } T_{\mathrm{if}}}{2 A_{2} Z_{3}\left(Z_{1}-Z_{2}\right)-A_{3} A_{4} Z_{1}^{2} T_{\mathrm{if}}+A_{1} A_{4} U_{\max } T_{\mathrm{if}} Z_{3}}
$$

and

$$
\lambda_{2}(T)=\frac{-Z_{1}^{3 / 2} Z_{3}^{-3 / 4} U_{\max }\left[2 Z_{3}^{1 / 2}+A_{4} T_{\mathrm{if}}\right]}{2 A_{2} Z_{3}\left(Z_{1}-Z_{2}\right)-A_{3} A_{4} Z_{1}^{2} T_{\mathrm{if}}+A_{1} A_{4} U_{\max } T_{\mathrm{if}} Z_{3}} ;
$$


and the optimal control in this region is:

$$
U^{\mathrm{op}}(T)=U_{\max }
$$

and this control remains optimal along the whole state space trajectory.

- (ii) If the final state $Z_{1}(T)$ and $Z_{2}(T)$, satisfies, $2 A_{2} Z_{3}\left(Z_{1}-Z_{2}\right)>A_{3} A_{4} Z_{1}^{2} T_{\text {if }}$ then the final values of the adjoint variables in this region are:

$$
\begin{aligned}
& \lambda_{1}(T)=0 \\
& \lambda_{2}(T)=0
\end{aligned}
$$

and the optimal control in this region is:

$$
U^{\mathrm{op}}(T)=0 \text {, }
$$

and this control remains optimal along the whole state space trajectory.

- (iii) If the final state $Z_{1}(T)$ and $Z_{2}(T)$, satisfies, $2 A_{2} Z_{3}\left(Z_{1}-Z_{2}\right)=A_{3} A_{4} Z_{1}^{2} T_{\text {if }}$ then the final values of the adjoint variables, $\lambda_{1}(T)$ and $\lambda_{2}(T)$, can hold any value. Switching points in these cases, may correspond to any state on the $U_{\min }$ - trajectory that crosses the point on the target curves that satisfy the above condition, and lies above the singular arc defined by $S=0, S$ being the singular function.

At the switching time $t_{\mathrm{s}}$, the adjoint variables $\lambda_{1}$, and $\lambda_{2}$, should hold the following values:

$$
\begin{aligned}
& \lambda_{1}\left(t_{\mathrm{s}}\right)=-\left.\frac{Z_{1}^{3 / 2}}{A_{1} Z_{3}^{7 / 4}}\right|_{t_{\mathrm{s}}}, \\
& \lambda_{2}\left(t_{\mathrm{s}}\right)=-\left.\frac{Z_{1}^{3 / 2}}{A_{1} Z_{3}^{7 / 4}}\left[1+\frac{A_{3} Z_{1}^{2}}{A_{2} Z_{3}^{1 / 2}\left(Z_{1}-Z_{2}\right)}\right]\right|_{t_{\mathrm{s}}} .
\end{aligned}
$$

The values of the adjoint variables at the final time $T$ are chosen such that the conditions (66) and (67) are satisfied. The optimal control switching in this case should be of the form:

$$
\begin{aligned}
& U_{\max } \rightarrow U_{\min } \quad \text { if } S<0 \text {. } \\
& U_{\min } \rightarrow U_{\max } \quad \text { if } S>0 \text {. } \\
& \left.\begin{array}{l}
U_{\max } \rightarrow U_{\mathrm{s}} \\
\text { or } \\
U_{\min } \leftrightarrow U_{\mathrm{s}}
\end{array}\right\} \quad \text { if } S=0 .
\end{aligned}
$$

In this case the singular function is given by:

$$
S=6 A_{2} Z_{3}^{3 / 2}\left(Z_{1}-Z_{2}\right)^{2}+10 A_{3} Z_{3} Z_{1}^{3}-14 A_{3} Z_{3} Z_{2} Z_{1}^{2}-5 A_{3} A_{4}\left(Z_{1}-Z_{2}\right) Z_{1}^{3} .
$$

\subsubsection{General cases, $C_{1}>0, C_{2}>0$}

At the final time $T$, the state and adjoint variables should satisfy the following conditions:

$$
\begin{aligned}
& Z_{1}(T)>T_{\text {if }}\left[1+A_{4}^{2} T_{\text {if }}^{2}\right]^{1 / 2}-A_{4} T_{\text {if }}^{2}, \quad \text { i.e. } Z_{1}(T)>Z_{2}(T), \\
& Z_{2}(T)=\frac{T_{\text {if }}}{2}\left\{\left[A_{4}^{2} T_{\text {if }}^{2}+4\left(1-A_{4} Z_{1}(T)\right)\right]^{1 / 2}-A_{4} T_{\text {if }}\right\}
\end{aligned}
$$

For the final values of the adjoint variables, we have two cases:

(i) If the final state satisfies the inequality:

$$
\frac{A_{4} Z_{1}^{3 / 2} C_{1} Z_{3}^{-3 / 4} T_{\text {if }}}{2 A_{2} Z_{3}\left(Z_{1}-Z_{2}\right)-A_{3} A_{4} Z_{1}^{2} T_{\text {if }}} \geq \frac{C_{2} Z_{1}^{3 / 2}}{A_{1} Z_{3}^{7 / 4}}
$$


then:

$$
\lambda_{1}(T)=\frac{-A_{4} Z_{1}^{3 / 2}\left(C_{1}+C_{2} U_{\max }\right) Z_{3}^{-3 / 4} T_{\mathrm{if}}}{2 A_{2} Z_{3}\left(Z_{1}-Z_{2}\right)-A_{3} A_{4} Z_{1}^{2} T_{\mathrm{if}}+A_{1} A_{4} U_{\max } T_{\mathrm{if}} Z_{3}},
$$

and

$$
\lambda_{2}(T)=\frac{-Z_{1}^{3 / 2}\left(C_{1}+C_{2} U_{\max }\right) Z_{3}^{-3 / 4}\left[2 Z_{3}^{1 / 2}+A_{4} T_{\mathrm{if}}\right]}{2 A_{2} Z_{3}\left(Z_{1}-Z_{2}\right)-A_{3} A_{4} Z_{1}^{2} T_{\mathrm{if}}+A_{1} A_{4} U_{\max } T_{\mathrm{if}} Z_{3}}
$$

and the final optimal control along this section of the target curve is:

$$
U^{\mathrm{op}}(T)=U_{\max }
$$

(ii) When the final state satisfies the inequality:

$$
\frac{A_{4} Z_{1}^{3 / 2} C_{1} Z_{3}^{-3 / 4} T_{\mathrm{if}}}{2 A_{2} Z_{3}\left(Z_{1}-Z_{2}\right)-A_{3} A_{4} Z_{1}^{2} T_{\mathrm{if}}} \leq \frac{C_{2} Z_{1}^{3 / 2}}{A_{1} Z_{3}^{7 / 4}}
$$

then:

$$
\lambda_{1}(T)=\frac{-A_{4} Z_{1}^{3 / 2} C_{1} Z_{3}^{-3 / 4} T_{\mathrm{if}}}{2 A_{2} Z_{3}\left(Z_{1}-Z_{2}\right)-A_{3} A_{4} Z_{1}^{2} T_{\mathrm{if}}}
$$

and

$$
\lambda_{2}(T)=\frac{-Z_{1}^{3 / 2} C_{1} Z_{3}^{-3 / 4}\left[2 Z_{3}^{1 / 2}+A_{4} T_{\mathrm{if}}\right]}{2 A_{2} Z_{3}\left(Z_{1}-Z_{2}\right)-A_{3} A_{4} Z_{1}^{2} T_{\mathrm{if}}}
$$

and the final optimal control along this section of the target curve is:

$$
U^{\mathrm{op}}(T)=0 .
$$

At the switching time $t_{\mathrm{s}}$, if any, the following conditions on the adjoint variables should hold:

$$
\begin{aligned}
& \lambda_{1}\left(t_{\mathrm{s}}\right)=-\left.\frac{C_{2} Z_{1}^{3 / 2}}{A_{1} Z_{3}^{7 / 4}}\right|_{t_{\mathrm{s}}} \\
& \lambda_{2}\left(t_{\mathrm{s}}\right)=-\left.\frac{C_{2} Z_{1}^{3 / 2}}{A_{1} Z_{3}^{7 / 4}}\left[1+\frac{A_{3} Z_{1}^{2}}{A_{2} Z_{3}^{1 / 2}\left(Z_{1}-Z_{2}\right)}+\frac{A_{1} C_{1} Z_{3}^{1 / 2}}{A_{2} C_{2}\left(Z_{1}-Z_{2}\right)}\right]\right|_{t_{\mathrm{s}}} .
\end{aligned}
$$

The optimal control switching in this case should be of the form:

$$
\begin{aligned}
& U_{\max } \rightarrow U_{\min } \quad \text { if } S<0 . \\
& U_{\min } \rightarrow U_{\max } \quad \text { if } S>0 \text {. } \\
& U_{\max } \leftrightarrow U_{\mathrm{s}} \\
& \text { or } \left.U_{\mathrm{s}}\right\} \text { if } S=0 \text {. } \\
& U_{\min } \leftrightarrow U_{\mathrm{s}}
\end{aligned}
$$



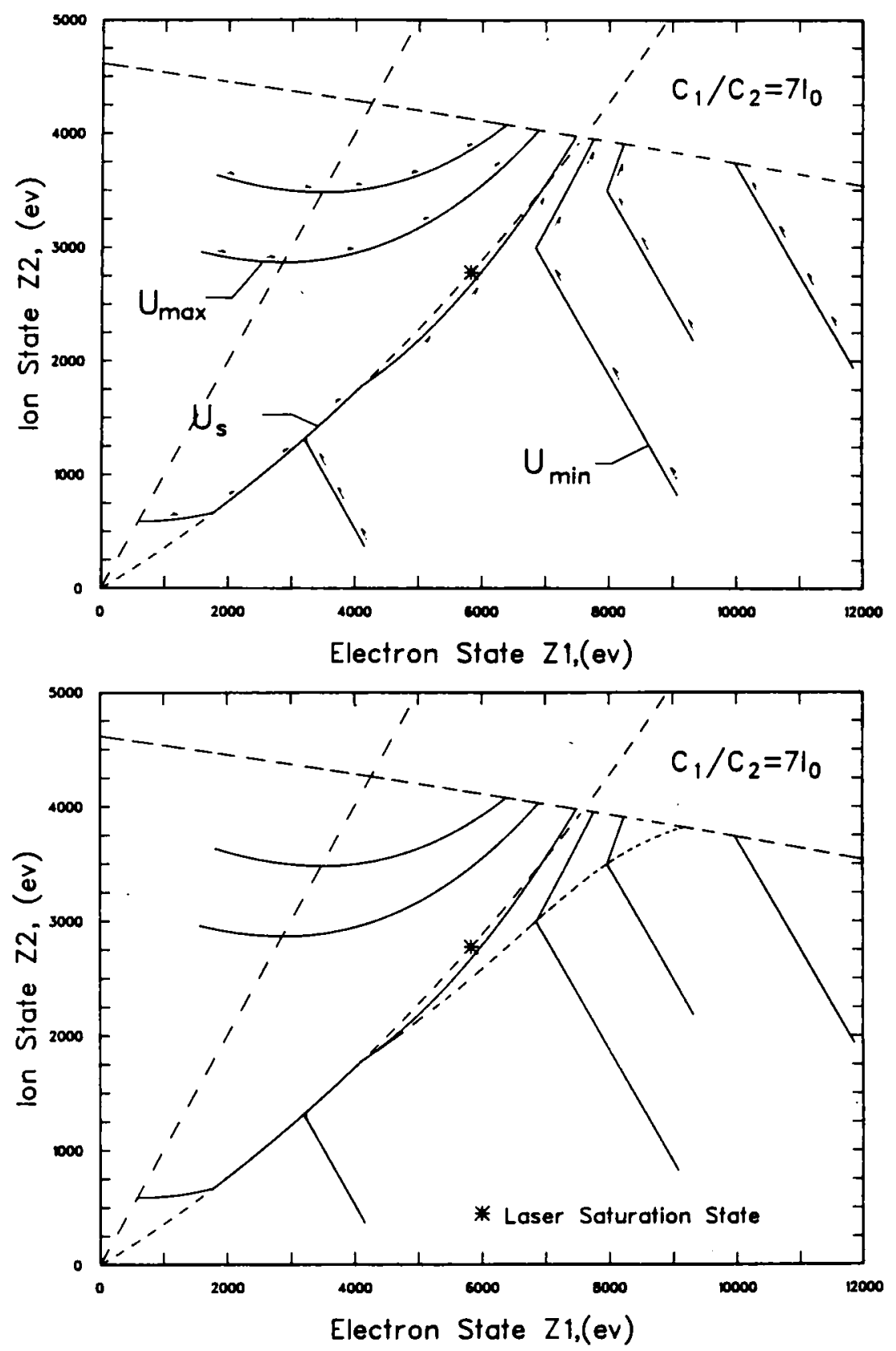

Fig. 7. Some optimal trajectories corresponding to a final ion temperature of $5 \mathrm{keV}$, for a value $C_{1} / C_{2}=7 U_{\max }$ and external magnetic field of $500 \mathrm{kG}$. 
The singular function $S$, is given by:

$$
\begin{aligned}
S\left(Z_{1}, Z_{2}, C_{1}, C_{2}\right)= & 6 A_{2} C_{2} Z_{3}^{3 / 2} Z_{1}^{2}-12 A_{2} C_{2} Z_{3}^{3 / 2} Z_{2} Z_{1}+6 A_{2} C_{2} Z_{3}^{3 / 2} Z_{2}^{2}+10 A_{3} C_{2} Z_{3} Z_{1}^{3} \\
& -14 A_{3} C_{2} Z_{3} Z_{2} Z_{1}^{2}+2 A_{1} C_{1} Z_{3}^{2} Z_{1}-6 A_{1} C_{1} Z_{3}^{2} Z_{2}+5 A_{1} A_{4} C_{1} Z_{3} Z_{1}\left(Z_{1}-Z_{2}\right) \\
& -5 A_{3} A_{4} C_{2}\left(Z_{1}-Z_{2}\right) Z_{1}^{3} .
\end{aligned}
$$

In the next section this set of conditions are implemented in order to obtain the switching curves.

\subsection{Switching curves}

In this section the necessary conditions that any optimal trajectory should satisfy at the final state are used to generate optimal backward trajectories that start on the target curve, in order to find the switching curves, i.e. the set of points in the phase plane where optimal control switchings occur. The switching point for each optimal backward trajectory is located where the optimal switching conditions summarized in the last section are satisfied.

Each backward trajectory was obtained by numerical integration of eqs. (24) through (27), using a simple corrector-predictor method.

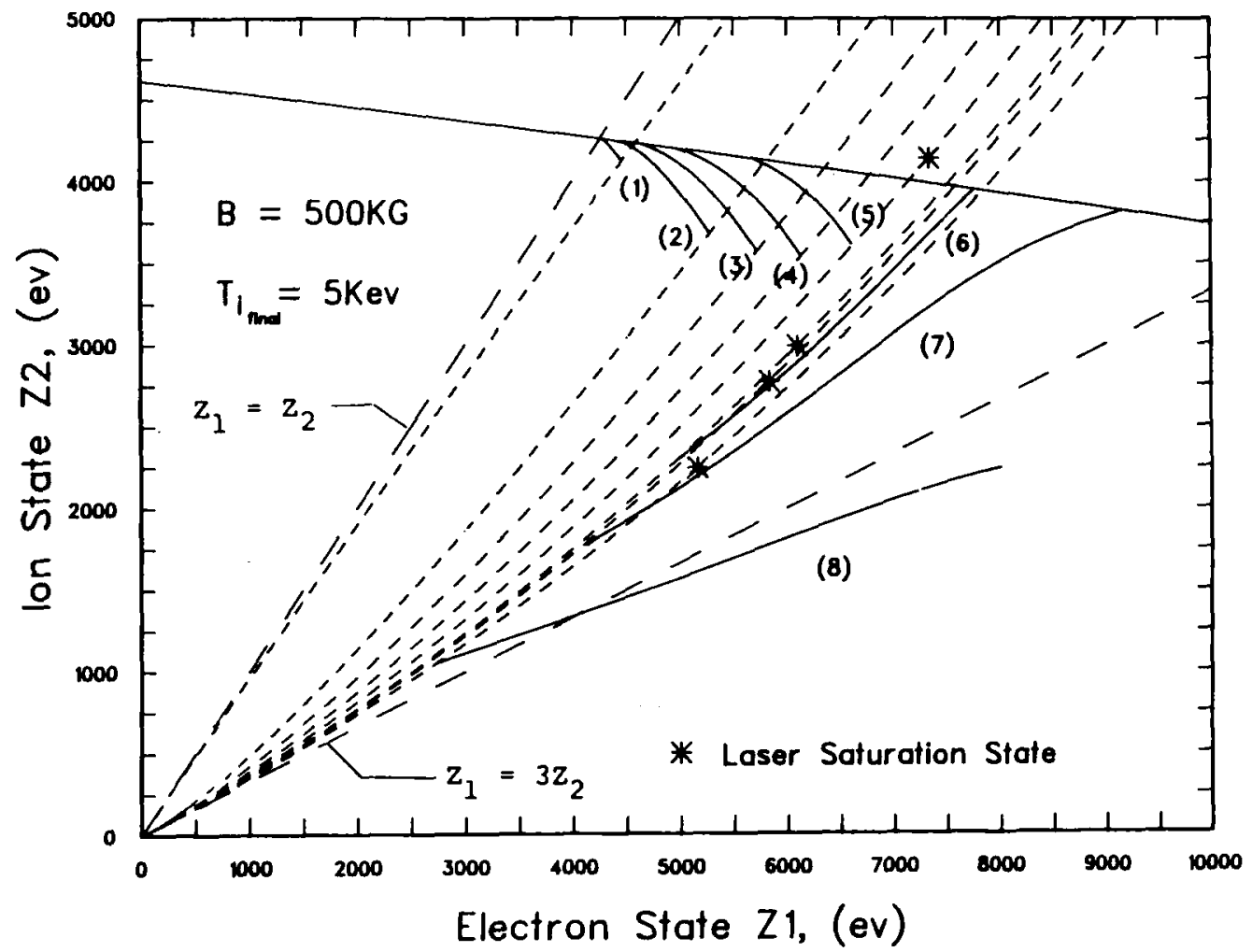

Fig. 8. Behavior of switching curves (continuous lines) given the final ion temperature of $5 \mathrm{keV}$, and an external magnetic field of 500 $\mathrm{kG}$. The dashed lines are the singular arcs. (1) $R=0$, (2) $R=1 / 5$, (3) $R=1 / 2$, (4) $R=1,(5) R=2$, (6) $R=5,(7) R=7$, (8) $R=$ infinite 

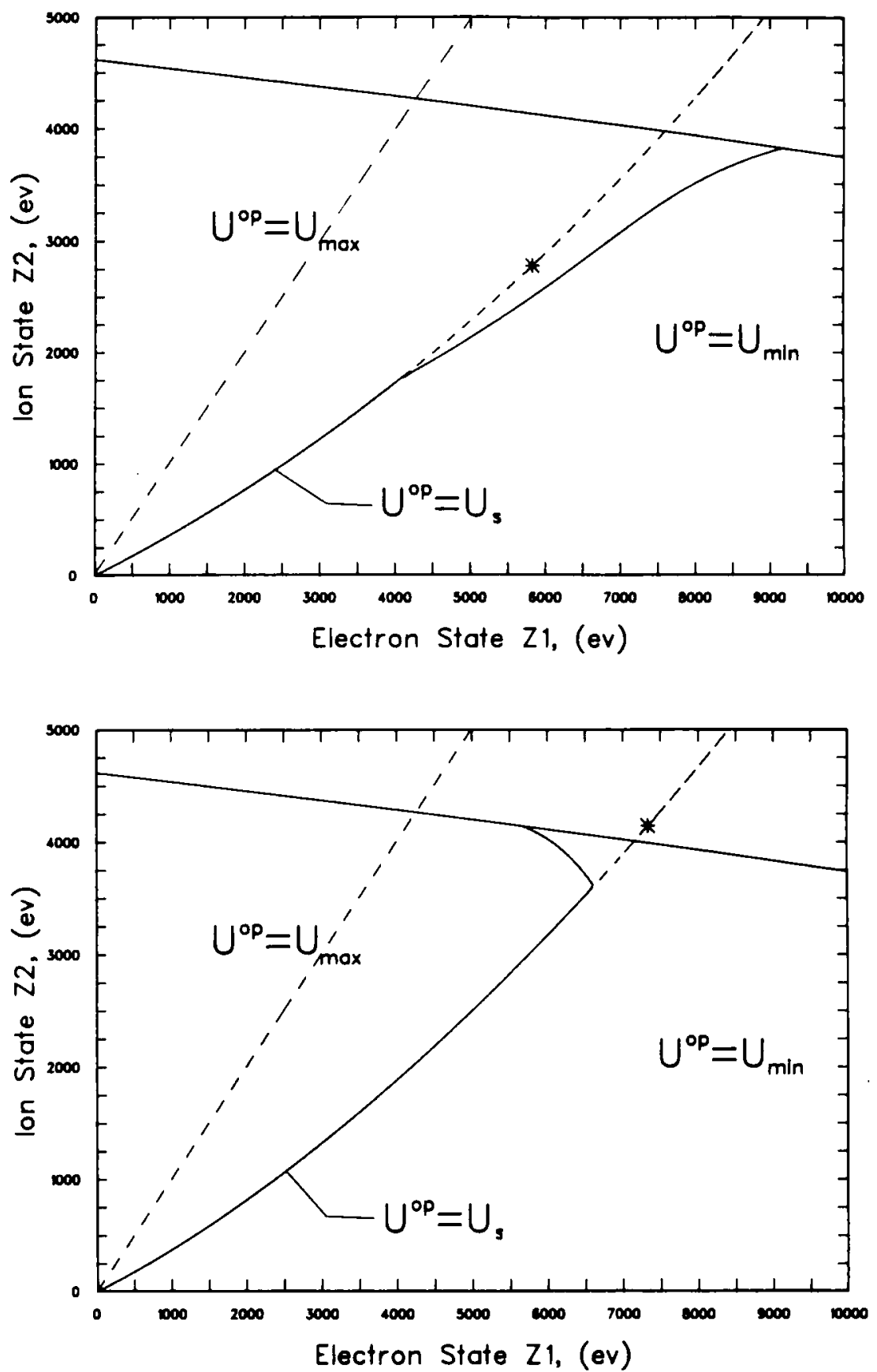

Fig. 9. Typical optimal control zones, corresponding to a final ion temperature of $5 \mathrm{keV}$ and external magnetic field of $500 \mathrm{kG}$. $C_{1} / C_{2}=7 U_{\max }$ (top), and $C_{1} / C_{2}=2 U_{\max }$ (bottom). 
The top graph in fig. 7 shows optimal trajectories for several initial states, final ion temperature of 5 $\mathrm{keV}$ and external magnetic field of $500 \mathrm{kG}$, that minimize the functional (19) with a ratio $C_{1} / C_{2}=7 U_{\max }$. The set of points in the phase plane where the optimal control switchings occur, form the "switching curve". These curves are shown in fig. 7 (bottom).

A fixed final ion temperature of $5 \mathrm{keV}$ and an arbitrary strong magnetic field of $500 \mathrm{kG}$ were chosen to illustrate the behavior of the switching curves when the weighting parameters $C_{1}$ and $C_{2}$ vary between the limiting cases of Minimum Energy Trajectories, $\left(C_{1}=0, C_{2}=1\right)$, and Minimum Time Trajectories $\left(C_{1}=\right.$ $1, C_{2}=0$ ). This behavior is shown in fig. 8 .

The objective of the switching curves is to separate the phase plane in regions in which a well defined optimal control is associated with each point of the phase plane.

In fig. 8 we can observe the transition between a switching curve corresponding to $U_{\max } \rightarrow U_{\min }$ transitions (which is located at the left hand side of the singular arc, and is (referred to as the "upper switching curve") and a switching curve located at the right hand side of the singular arc, which corresponds to transitions of the type $U_{\min } \rightarrow U_{\max }$, (referred to as “lower switching curve").

The optimal control associated with each state of the phase plane when only the upper switching curve or the lower switching curve exists, is shown in fig. 9.

When the intersection point between the upper switching curve and the singular arc, lies above the laser saturation state, then a lower switching curve exists, which ends below the laser saturation state, unfortunately these curves are not observed in fig. 8, due to the set of weighting parameters used in the calculations.

It is observed from figs. 8 and 9 , that in the region $Z_{1}<Z_{2}$, the optimal control is always $U_{\max }$; this is reasonable since in this region there is no ion heating. On the other hand if the electron state variable is larger that the ion'state variable in some cases the optimal control strategy is just to leave the electron and the ion temperatures to relax, i.e with $U^{\text {op }}=0$.

In the next section the implementation of the optimal control strategies described in this section, using the optimal switching curves obtained are illustrated.

\section{Optimal trajectories}

In this section typical optimal trajectories are obtained for several ratios $C_{1} / C_{2}$ of the weighting parameters, following the optimal control strategy presented in the last section.

An initial state corresponding to a temperature of $10 \mathrm{eV}$ for both ions and electrons and a final ion temperature of $5 \mathrm{keV}$ were used in all the following cases in order to compare the total energy spent and the heating time in the process, when different sets of weighting parameters are used. We should keep in mind, however, that once the switching curves have been determined, the optimal control for any state is available.

The sets of weighting parameters that were used correspond to those utilized to obtain the switching curves in fig. 8.

When the optimal control strategies discussed in the last section were implemented, the optimal trajectories in the phase plane shown in fig. 10 were obtained. Due to the initial state used, all the trajectories contain a singular subarc.

As it was mentioned before in section 3.4, the Minimum Energy trajectories keep the electron state variable, as small as possible, increasing the coupling between the laser beam and the electrons and reducing the bremsstrahlung radiation. As we can deduce from eq. (48), if no bremsstrahlung radiation were included $\left(A_{3}=0\right)$ the singular arc for the case of Minimum Energy Trajectories, reduces to the straight line $Z_{1}=Z_{2}$; the reason is that, since no energy losses exist, the heating time may grow indefinitely without affecting the penalty function; thus reducing the electron temperature increases the 


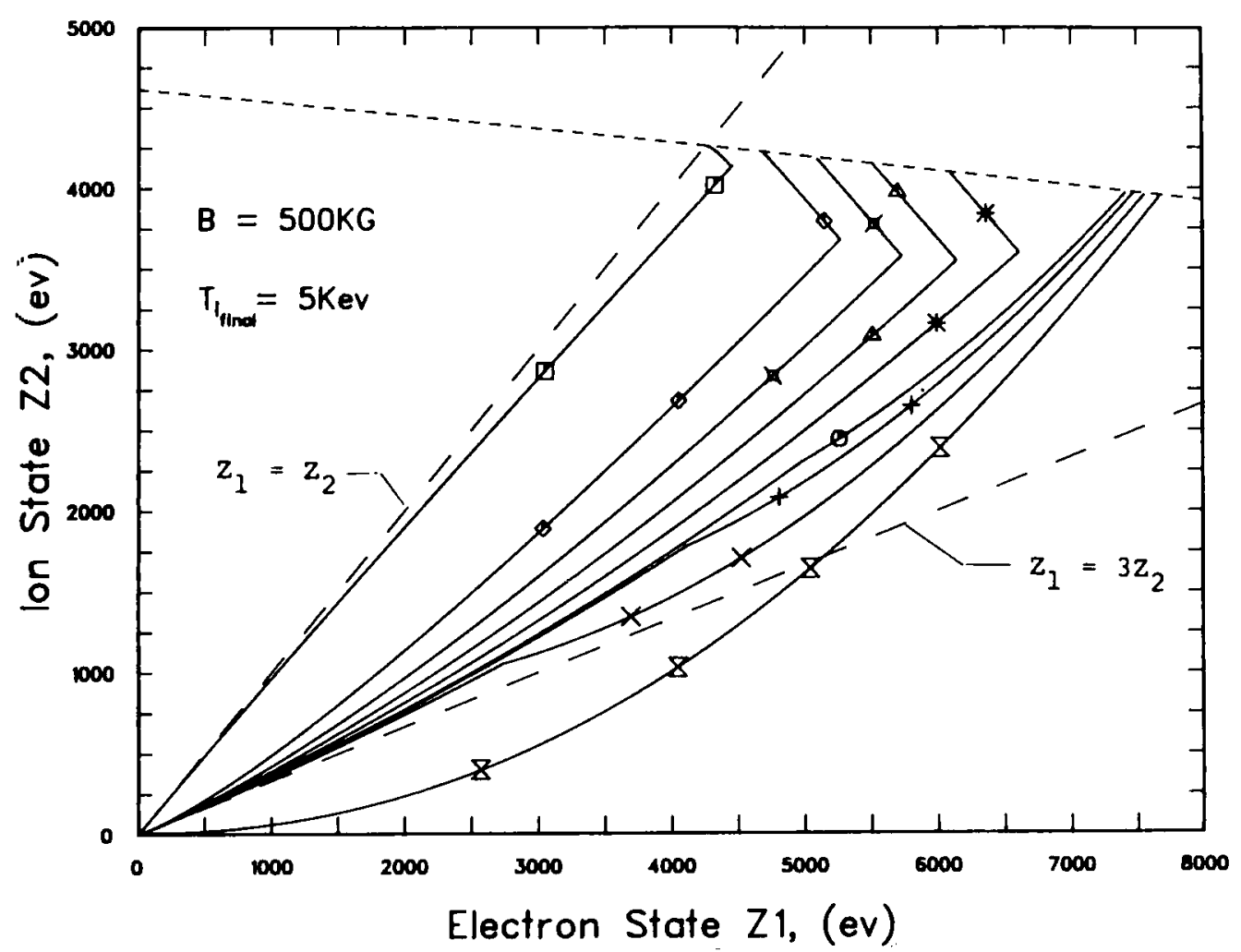

Fig. 10. Behavior of optimal trajectories with initial state of $10 \mathrm{eV}$ (ions and electrons) and final ion temperature of $5 \mathrm{keV}$. $\square R=0$, $\diamond R=1 / 5, \bigotimes R=1 / 2, \Delta R=1, * R=2, \bigcirc R=5,+R=7, \times R=$ infinite, 8 Const. laser pulse, $R=C_{1} / C_{2} \times I_{0}$.

efficiency in the absorption of the laser energy by the electrons, however the energy transfer rate between electrons and ions in the plasma becomes infinitesimally small.

Minimum Time Trajectories, on the other hand, keep the electron temperature and the ion temperature along the singular subarc satisfying the relation (47); this maximizes the energy transfer rate between ions and electrons; in the same way as in the constant density case [12], the exit point from the singular arc appears below the laser saturation state.

The other trajectories constitute a compromise between the energy spent and the heating time, with different weighting coefficients.

The optimal laser intensity profiles that drove the plasma from the initial state to the final state along the optimal trajectories in fig. 10 are plotted as a function of time in figs. 11 through 13.

Table 1, shows the control switching times, the heating time and the total energy spent in the heating process for the different ratios $C_{1} / C_{2}$ used in the calculations.

Fig. 14 shows the behavior of the logarithm of the heating time in microseconds and the fraction of the energy spent in the heating process compared with that used when a constant laser pulse is utilized, both plotted against the ratio $C_{1} /\left(C_{2} \times I_{0}\right)$. A possible use of these figures can be illustrated as follows: Suppose the initial state of the plasma is $10 \mathrm{eV}$, for both ions and electrons, and the maximum energy available and maximum laser intensity $I_{0}$ have been determined for the laser system being used, the maximum value of the ratio $C_{1} / C_{2}$ can then be determined from fig. 14 (top); given this value of $C_{1} / C_{2}$, the minimum heating time available, with the given the total energy constraint, can be determined, fig. 14 

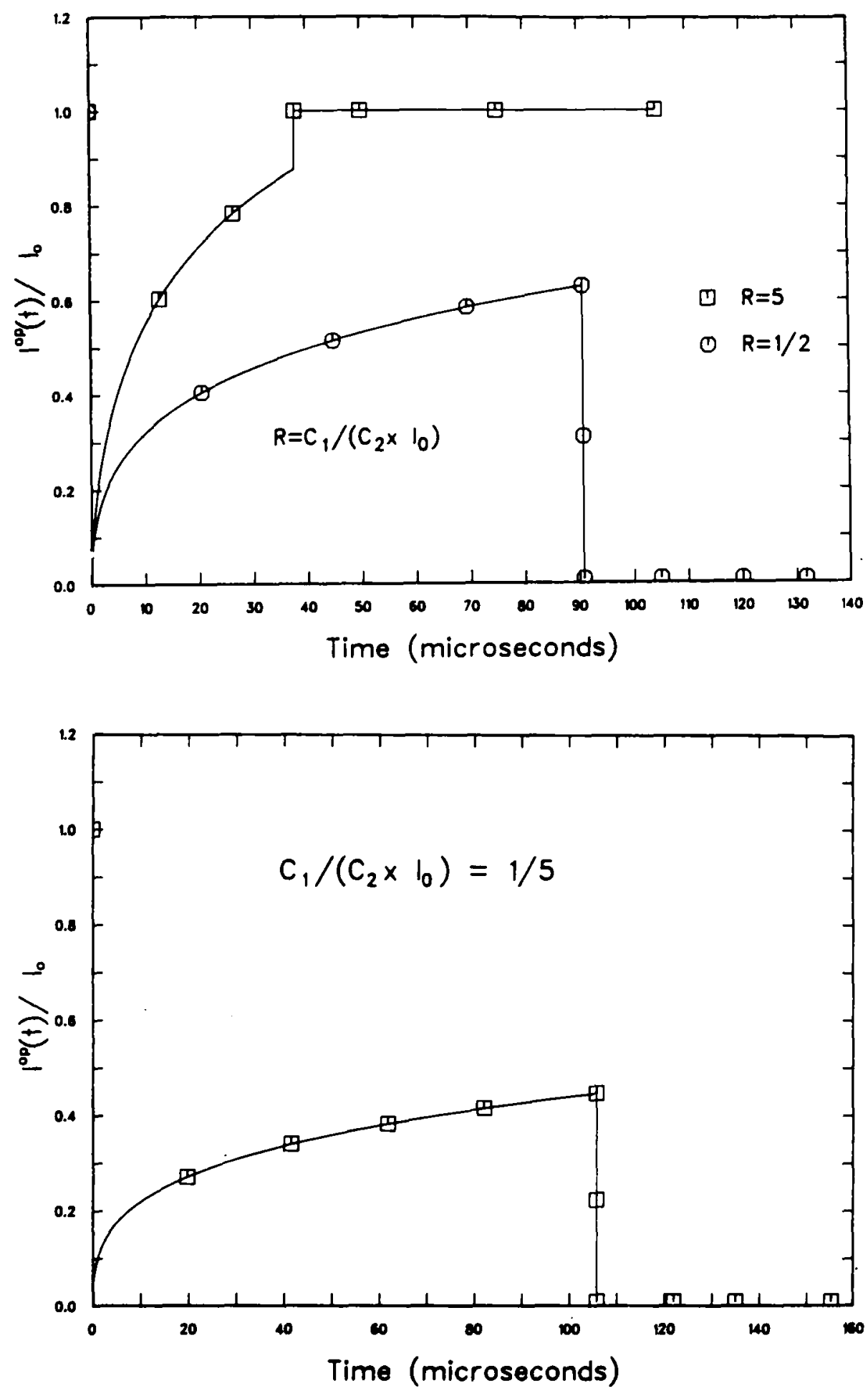

Fig. 11. Optimal laser pulse for several ratios of the weighting parameters. 

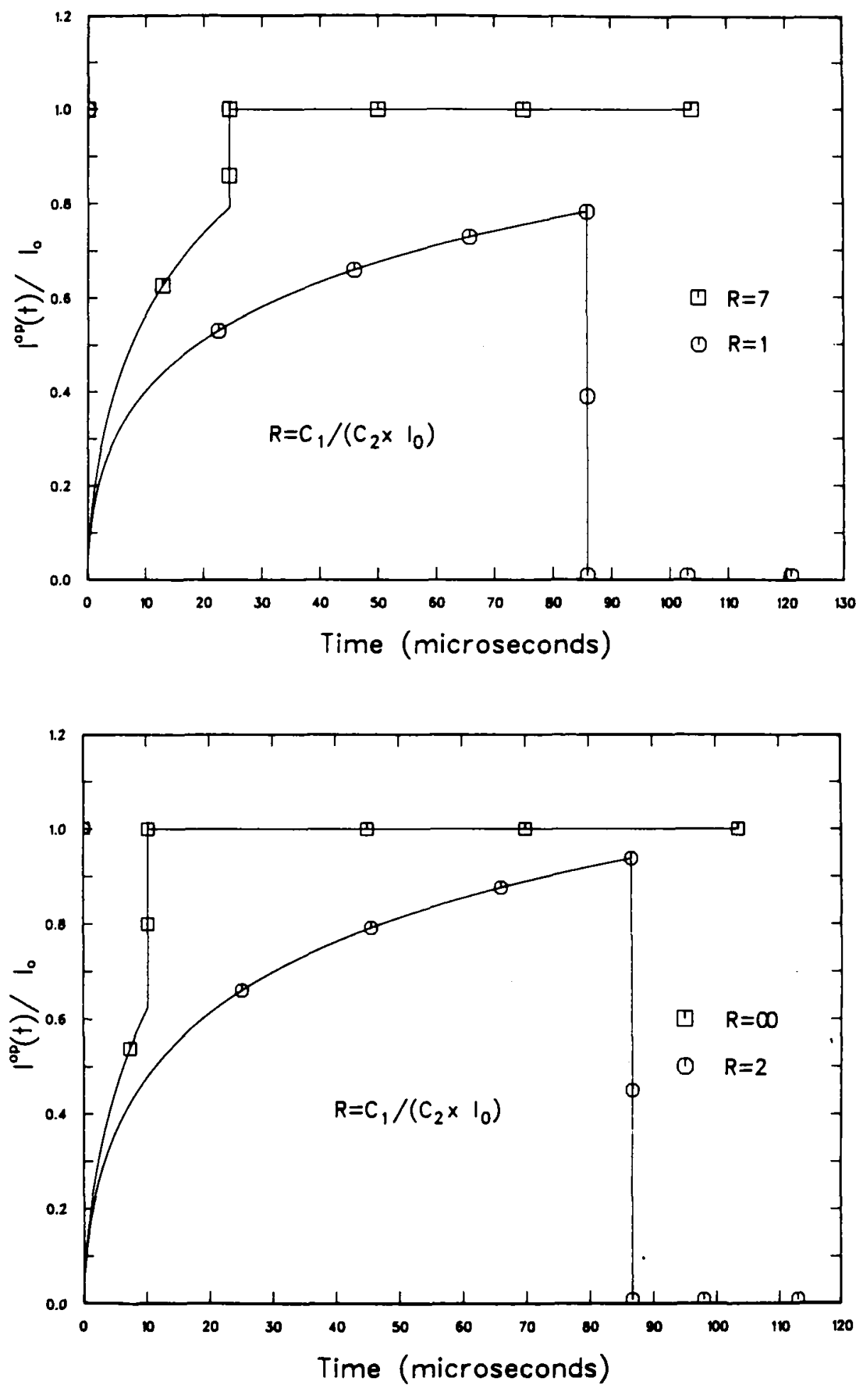

Fig. 12. Optimal laser pulse for several ratios of the weighting parameters. 


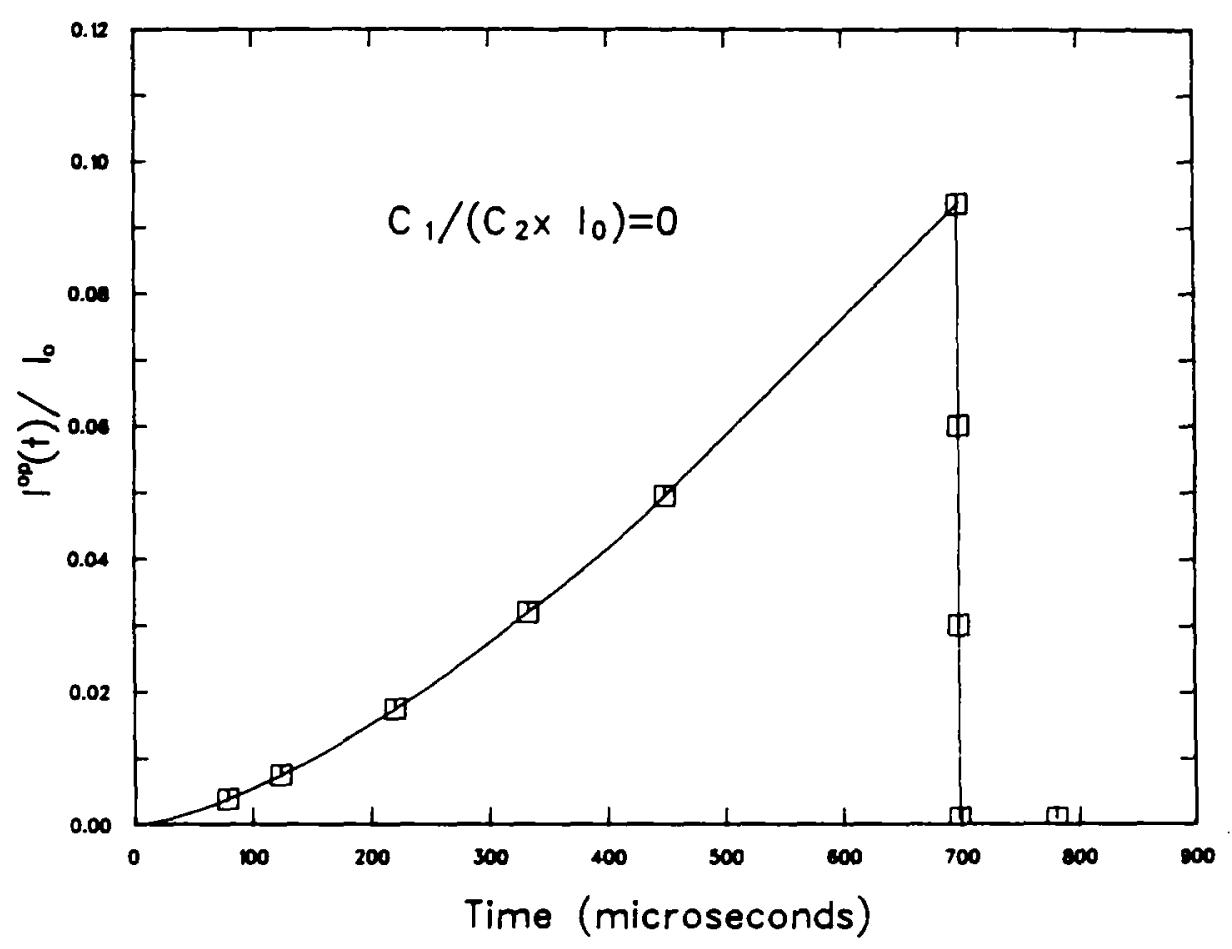

Fig. 13. Optimal laser pulse for Minimum Energy Trajectory.

(bottom). If smaller values of $C_{1} / C_{2}$ are chosen, then, the heating time will be larger, however, the energy necessary in the heating process will be smaller.

Comparing our results with those obtained by Vagners et al. [1] for a maximum total energy of $14.37 \times 10^{25} \mathrm{eV} / \mathrm{cm}^{2}$, they got a heating time of $128.3 \mu \mathrm{s}$, as compared with that of $\sim 113 \mu \mathrm{s}$, deduced from table 1. Also, the optimal shape of the laser pulse is different, the reason lies in part, in the inclusion of bremsstrahlung losses in the electron energy conservation equations; so that, energy transfer rate from electrons to ions have to be accelerated in order to minimize these losses; and on the other hand, they used $\frac{3}{2} n \mathrm{~d} T_{\mathrm{e}} / \mathrm{d} t$ instead of $\frac{3}{2} \mathrm{~d} / \mathrm{d} t\left(n T_{\mathrm{e}}\right)$ in eq. (1).

Table 1

Heating data corresponding to optimal trajectories in fig. 10

\begin{tabular}{lllllll}
\hline$C_{1}$ & $\begin{array}{l}\text { Switch. time } \\
C_{2} I_{0}\end{array}$ & $\begin{array}{l}\text { Switch. time } \\
U_{\mathrm{max}} \rightarrow U_{\mathrm{s}}\end{array}$ & $\begin{array}{l}\text { Switch. time } \\
U_{\mathrm{s}} \rightarrow U_{\min }\end{array}$ & $\begin{array}{l}\text { Switch. time } \\
U_{\max } \rightarrow U_{\min }\end{array}$ & $\begin{array}{l}\text { Heating } \\
\text { time }\end{array}$ & $\begin{array}{l}\text { Heat. energy } \\
\left(\times 10^{25} \mathrm{eV} / \mathrm{cm}^{2}\right)\end{array}$ \\
\hline 0 & $0.02 \mathrm{ps}$ & - & $698.03 \mu \mathrm{s}$ & - & $780.53 \mu \mathrm{s}$ & 5.686 \\
$1 / 5$ & $28.3 \mathrm{ps}$ & - & $105.85 \mu \mathrm{s}$ & - & $155.25 \mu \mathrm{s}$ & 8.204 \\
$1 / 2$ & $29.3 \mathrm{ps}$ & - & $90.78 \mu \mathrm{s}$ & - & $131.75 \mu \mathrm{s}$ & 9.883 \\
1 & $30.6 \mathrm{ps}$ & - & $85.91 \mu \mathrm{s}$ & - & $120.78 \mu \mathrm{s}$ & 11.67 \\
2 & $29.6 \mathrm{ps}$ & - & $86.70 \mu \mathrm{s}$ & - & $113.10 \mu \mathrm{s}$ & 14.14 \\
5 & $31.1 \mathrm{ps}$ & $37.89 \mu \mathrm{s}$ & - & - & $104.30 \mu \mathrm{s}$ & 20.43 \\
7 & $31.1 \mathrm{ps}$ & $24.43 \mu \mathrm{s}$ & - & - & $103.88 \mu \mathrm{s}$ & 20.97 \\
infinite & $31.2 \mathrm{ps}$ & $10.34 \mu \mathrm{s}$ & - & - & $103.35 \mu \mathrm{s}$ & 21.94 \\
Const. pulse & - & - & - & - & $105.79 \mu \mathrm{s}$ & 23.80 \\
\hline
\end{tabular}



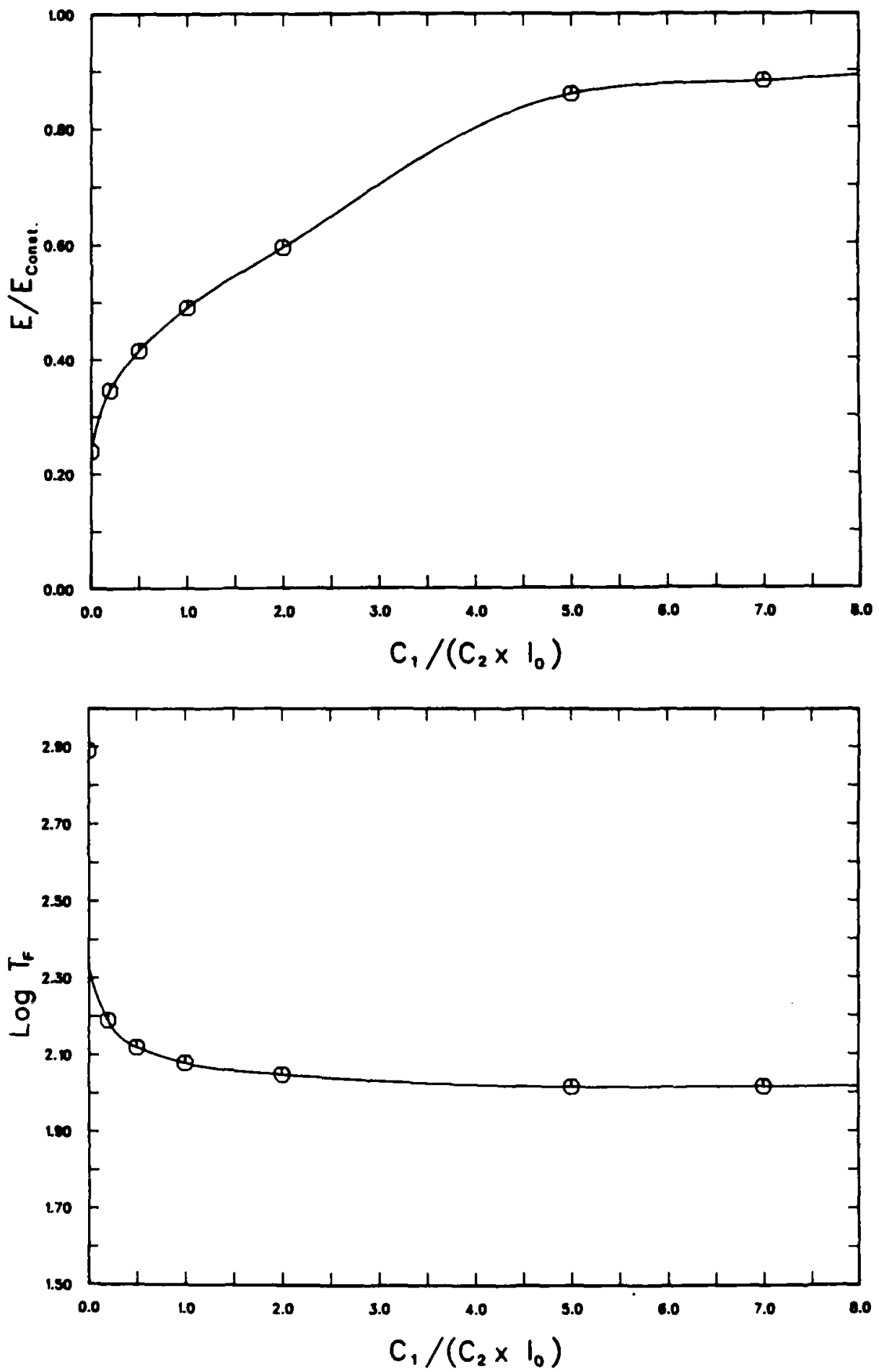

Fig. 14. Fraction of the energy spent using a constant laser pulse, and the logarithm of the heating time (in microseconds) against the ratio of the weighting parameters. 


\section{Conclusions}

We have considered the laser-heating of plasmas confined in strong solenoidal magnetic fields. Optimal laser heating strategies where obtained using optimal control theory; the optimization calculations where carried out to the end by developing a "Flooding" technique.

We have shown, within the limitations of the plasma model used here, that considerable savings can be achieved in the laser energy requirement by resorting to laser intensity optimization. For example, by appropriately shaping the laser pulse, the energy required in the heating process may be reduced to around $25 \%$ of the energy necessary when a square pulse is used; however in this case the heating times may grow as high as $700 \%$ yielding relatively large heating times. Similarly to the constant density case [12,13], minimum time trajectories may not yield a significant reduction in heating time as compared with the use of a square pulse.

Extensions to more ambitious plasma models are certainly desirable, we hope this work will stimulate further applications of the optimization theory in this direction.

\section{Acknowledgments}

One of the authors (J.V.E.) wishes to express his gratitude to the members of the Nuclear Engineering Department at the University of Michigan for their hospitality and assistance during the course of this research. He also wishes to gratefully acknowledge the financial support provided by Consejo Nacional de Ciencia y Tecnología (CONACyT), the National Science Foundation and the Michigan Memorial Phoenix Project.

\section{References}

[1] J. Vagners, R.D. Neal and G.C. Vlases, Phys. Fluids 18 (1975) 1374.

[2] A.E. Bryson Jr. and Y.C. Ho, Applied Optimal Control (Hemisphere Publ. Co., New York, 1975).

[3] L.C. Steinhauer and H.C. Ahlstrom, Phys. Fluids 18 (1975) 541.

[4] S.Y. Yuen, B. Lax and D.R. Cohn, Phys. Fluids 5 (1975) 829.

[5] J. Dawson and C. Oberman, Phys. Fluids 5 (1962) 517.

[6] E.H. Holt and R.E. Haskell, Foundations of Plasma Dynamics (MacMillan Co., New York, 1965).

[7] S. Glasstone and R.H. Lovberg, Controlled Thermonuclear Reactions (Krierger Publ. Co., New York, 1975).

[8] S. Chandrasekhar, Plasma Physics (Univ, of Chicago Press, 1962).

[9] R. Gabasov and F.M. Kirillova, SIAM J. of Control 10 (1972) 127.

[10] C.D. Johnson, Adv. in Control Systems, Vol. 2 (Academic Press, New York, 1965).

[11] H.J. Kelley, R.E. Kopp and A.G. Moyer, Topics in Optimization, Vol. 2 (Academic Press, New York, 1966).

[12] J. Vitela, Ph.D. Thesis, University of Michigan (1984).

[13] J. Vitela E. and A.Z. Akcasu, J. Optimization, Theory and Applicatiuns, Vol. 52, No. 1 (1987). 